
\title{
Resesarch Sulure \\ Evidences of Intra-Group Orca Call Rate Modulation Using A Small-Aperture Four Hydrophone Array
}

Marion Poupard ( $\triangle$ marion.poupard@lis-lab.fr)

Universite De Toulon Et Du Var

Helena Symonds

Pacific Orca Society/OrcaLab

Paul Spong

Pacific Orca Society/OrcaLab

Hervé Glotin

Universite De Toulon Et Du Var

\section{Research Article}

Keywords: Orcinus orca, Call Rate, Bonferroni

Posted Date: December 9th, 2020

DOI: https://doi.org/10.21203/rs.3.rs-116685/v1

License: (c) (1) This work is licensed under a Creative Commons Attribution 4.0 International License.

Read Full License 


\title{
Evidences of Intra-group Orca Call Rate Modulation using a Small-aperture Four Hydrophone Array
}

\author{
Marion Poupard ${ }^{1,3}{ }^{*}$, Helena Symonds ${ }^{2}$, Paul Spong ${ }^{2}$, and Hervé Glotin ${ }^{1,4}$ \\ ${ }^{1}$ Université de Toulon, Aix Marseille Univ., CNRS, LIS, DYNI, Marseille, France \\ ${ }^{2}$ Pacific Orca Society/OrcaLab, Canada \\ ${ }^{3}$ BIOSONG, SAS, France \\ ${ }^{4}$ Université de Toulon, INPS, SMloT, France \\ *marion.poupard@lis-lab.fr, helena@orcalab.org, glotin@univ-tln.fr
}

\begin{abstract}
Acoustic emissions are vital to orcas (Orcinus orca) to socialize, hunt, orient, and maintain spatial awareness. In order to better analyze their inter and intra-group communication, we propose a novel protocol that allows us to associate vocalizations with their emitter (individual/matriline). Our approach is based on a low cost small-aperture four hydrophone array fixed near the shore up to a few km away from the orcas' path, operated in conjunction with visual identification. It was conducted in the summer of 2019 off northern Vancouver Island, Canada, at the research station OrcaLab. A total of 722 calls were extracted and localized in azimuth via the hydrophone array from 3 case studies in which different events took place.

We then calculated the Call Rate (CR) for each individual/matriline in order to describe their acoustic activity. Results show that $\mathrm{CR}$ is modulated according to the distance of the signaler from the joint group, the presence of another group, and the anthropic pressure (nearby cruise ship). This shows evidence of intertwined calls. This protocol does not interfere with the animals and opens new perspectives towards inter and intra-group communication analysis.
\end{abstract}

\section{Introduction}

Orca (Orcinus orca) is a marine mammal that inhabits oceans across globe. For orcas, the ability to emit sound, hear and vocalize is vital to performing activities such as communication, reproduction and hunting ${ }^{1,2}$. They can produce a wide variety of sounds including clicks, whistles, and pulsed calls ${ }^{3}$. Pulsed calls or vocalizations are primordial for communication, and a pod can share between 7-17 different calls classed by types ${ }^{4}$. Vocal activity also depends on the group's activity ${ }^{5}$. Numerous acoustic studies have already proven that pulsed vocalization plays an important role in the social life of the group ${ }^{5-7}$. This study focuses on the Northern Resident Killer Whale (NRKW) community composed of different pods and matrilines ${ }^{8}$. Three distinct ecotypes of orcas live in the waters of British Columbia (BC) : Resident, Transient (Bigg's) and Offshore ${ }^{9}$. Transient orcas are marine mammal-eaters whereas Residents hunt mostly salmon ${ }^{10}$. Offshore orcas eat principally halibut (Hippoglossus s.) and shark $^{11}$.

There are two communities in coastal BC, the Southern Resident community and Northern Resident community (NRKW).

The NRKW is composed of 15 different pods and inhabits the waters between Vancouver Island and Southeast Alaska. Pods are groups of related orcas that frequently live and travel together. They also share the same acoustic dialect. The smallest unit of socialization within the pods is the matriline, which links individuals through maternal descent ${ }^{12}$.

In order to grasp the richness of orcas' intra-group communication, it is necessary to analyze the acoustic production of each individual or matriline during natural activities in the wild.

Most studies so far describe pod level call behavior ${ }^{13,14}$. It has proved challenging to demonstrate vocalizations produced by an observed matriline or individual.

Therefore it seems useful to put in place methods and protocols that associate vocalizations with an individual or matriline. Many works have described the vocal exchanges of animals as the response of a receiver after the emission of a particular signal $^{15,16}$. Some studies have already identified the signaler in a group of orcas ${ }^{13}$, finding that the types and timing of calls are influenced by the calling behaviors of other members of the group.

Miller ${ }^{17}$ used an array with 15 hydrophones towed from a boat. Orcas are easily disturbed ${ }^{18}$ so it is possible that the results from this study could have been influenced by the presence of the boat. As we will see in our results below, the CR change depending on whether there is a boat present or not.

Moreover, the deployment of a towed array is costly and could produce a limited amount of noisy data; it is therefore not convenient for a long term natural experiment on free ranging whales.

In this study using our localization method allowed us to accurately assign an azimuth to a surface entity. 
Once the individuals/matrilines were identified we used a quantitative variable to describe the vocal behaviour of the orcas : the Call Rate (CR), equal to the number of vocalizations each $10 \mathrm{~s}$ for each individual/matriline. The CR is commonly used in underwater ${ }^{19,20}$ and terrestrial ${ }^{21,22}$ bioacoustics to quantitatively describe the emission of sound signals.

The objective of our research was to proceed with a novel protocol that allowed us to acoustically identify individual/matriline vocalizations in order to study the $\mathrm{CR}$ under certain conditions.

This was done by using a low-cost hydrophone array, easily deployed in various sites, equipped with high quality call separation. We employed a novel audio-visual protocol based on the visual identification of orcas from the shore and a four-hydrophone array submerged nearby offshore to separate each of the calls being emitted several hundred meters away. In our study we will present 3 different case studies to validate our protocol.

The section Material and Methods, present the recording station and annotation methods. Then, we explain the computing of the azimuth of the calls, and the CR analysis. We applied this protocol on 722 calls taken from 3 cases in which different events took place. Finally, we summarize the results and discuss them.

\section{Results}

The sessions of this summer totaled 42 passages of orcas with vocalizations and 29 passages of orcas without vocalizations (Tab.1).

Table 1. Summary of the records of all of the sessions.

\begin{tabular}{|c|c|c|c|c|c|c|c|}
\hline Session & $\begin{array}{c}\text { Start } \\
(\mathbf{d d} / \mathbf{m m})\end{array}$ & $\begin{array}{c}\text { End } \\
(\mathbf{d d} / \mathbf{m m})\end{array}$ & $\begin{array}{c}\text { Cumul. Time } \\
(\mathbf{h h}: \mathbf{m m})\end{array}$ & Cumul. Day & Vocal Passage & Non Vocal Passage & Total of the passages \\
\hline 1 & $20 / 07$ & $01 / 08$ & $17: 40$ & 11 & 10 & 9 & 19 \\
\hline 2 & $04 / 08$ & $22 / 08$ & $17: 30$ & 17 & 12 & 6 & 18 \\
\hline 3 & $23 / 08$ & $15 / 08$ & $17: 40$ & 11 & 19 & 15 & 34 \\
\hline Total & & & $52: 50$ & 39 & 42 & 29 & 71 \\
\hline
\end{tabular}

Within the 71 passages, 57 passages were during daytime and 29 passages included vocalizations. We are able to isolate calls for three individuals (three males), because the males were somewhat removed from the group (minimum 50m). A total of 85 vocalizations were produced by these three males (Tab.2).

Table 2. Summary of the 3 individuals for which we isolated calls. A66 appeared twice.

\begin{tabular}{|r|r|r|r|r|}
\hline Date & $\begin{array}{r}\text { Pod } \\
\text { Id. }\end{array}$ & $\begin{array}{r}\text { Matriline } \\
\text { Id. }\end{array}$ & $\begin{array}{r}\text { Individual } \\
\text { Id. }\end{array}$ & $\begin{array}{r}\text { Call Count } \\
\text { (85 in Tot.) }\end{array}$ \\
\hline $04 / 09$ & A05 & A42 & A66 & 16 \\
\hline $31 / 08$ & I11 & I4 & I76 & 24 \\
\hline $22 / 07$ & A05 & A23 & A60 & 19 \\
\hline $30 / 08$ & A05 & A42 & A66 & 26 \\
\hline
\end{tabular}

We focused on three particular events : $11^{\text {th }}, 30^{\text {th }}$ and $31^{\text {st }}$ of August. The Fig.1 lists all the vocalizations heard and annotated. The color for each call corresponds to the color in the scatter plot in Fig.4 8,9 and 13. 


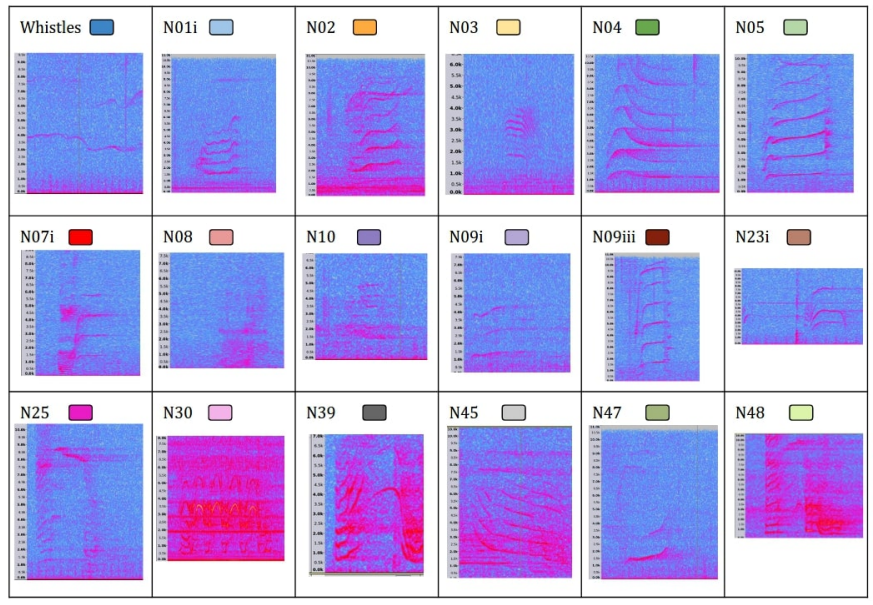

Figure 1. List of the calls annotated in each experiment.

\section{First observation ( $11^{\text {th }}$ off August)}

The first case study was on the $11^{\text {th }}$ of August, when a group made up of the A25, A50, A54, and I04 matrilines (mat.) came through (BP) at 4:46pm.

During this passage, the orcas were calm, and moved at a moderate swim velocity, but at 5:08:20pm a cruise ship (Fig.2) passed in front (OL) (traveling in the same direction as the orcas). Once the boat was present, the group dispersed and several orcas surfed in its wake. This made individual identification difficult.

During the first part (until 5:15pm) of the track, it was possible to recognize eight individuals from A05 and A01 pods (Fig.2). After the passage of the boat, the orcas split up so we lost the identification of four whales. From 5:16pm to 5:30pm we had the identifications of A61 (mat.A25), A72 (mat. A50), A108 (mat. A50) and I76 (mat. I04). Visual observations were supplemented by acoustic analyses. From 4:56pm to 5:10pm the orcas were together in BP traveling at a slow speed. When the cruise ship arrived, the whales started to accelerate and followed the boat until azimuth $100^{\circ}$. We can see this fast track visually and acoustically from 5:06pm to 5:15pm. During the last part of the track, from 5:23pm to 5:33pm, we observed that some orcas continued their trajectory while four individuals (from three different matrilines) turned around : A61 (mat.A25), A72 (mat.A50), A108 (mat.A50) and I76 (mat.I04). The male A61 (orange) is high in azimuth. Individuals A72, A108 and I76 had been travelling together for ten minutes.

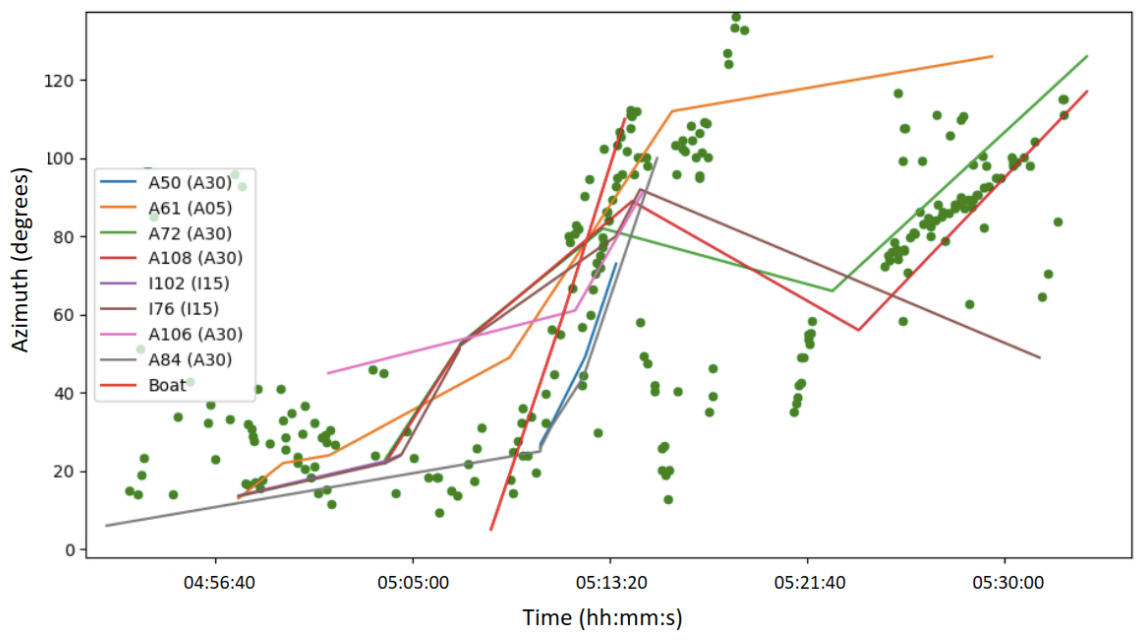

Figure 2. Graph of the passage of the $11^{\text {th }}$ of August. Each green point represents a vocalization placed in azimuth. The different curves are the positions of each individual visually detected.

The Fig. 3 presents the trajectories of a different group during the $11^{\text {th }}$ of August and the associated pictures. We noticed that at the start of the trajectory, the orcas were gathered in a single group (picture on the left). Then different smaller groups 
formed and some orcas were on the right and left of the boat (picture 2 and 3). During the end of the track some individuals "surfed" behind the ship (picture 4). This visual observation confirmed our acoustic position.

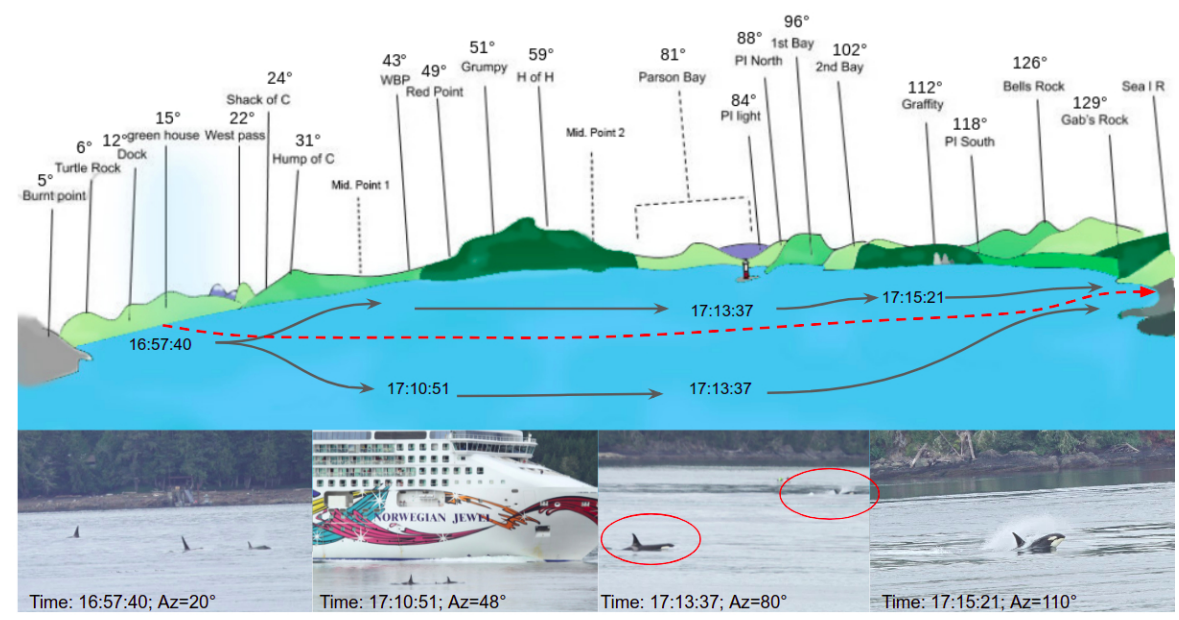

Figure 3. Graph of the passage of the $11^{\text {th }}$ of August. Top : representation of the view of BP from OL with the visual position of the group.The red line is the boat trajectory. Bottom : pictures taken from OL deck.

We were able to extract the types of vocalizations (Fig.4). We divided this track in 6 parts. The first and second parts were before the arrival of the cruise ship when the orcas travelled slowly and emitted call types N25, N04, N02, NO9iii. The third part happened when the boat came through BP and the orcas followed it (surfing) and produced only N04, N47 calls (1700 to $2000 \mathrm{~s}$ ). At the end of the boat passage (period 4), they produced a lot of N04 and N03 calls, and some of them turned around. During period 5 and 6, from 2700 to $3200 \mathrm{~s}$, the track from $90^{\circ}$ to $120^{\circ}$ is composed of N04, N47 calls. This is when the individuals, A72, A108 and I76 were travelling very close to each other. According to these observations, we can suppose that the boat not only had an influence on the velocity of the orcas, but also on the type of calls used.

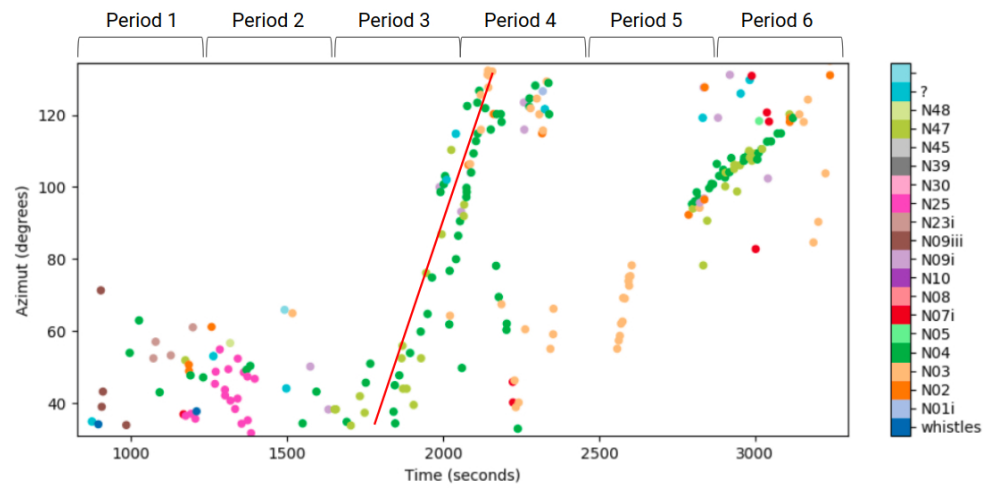

Figure 4. Graph of the passage of the $11^{\text {th }}$ of August. Each point represents a vocalization placed in azimuth. Each color represents a type of call. The most common types are N04, N03, N48. The red line is the visual trajectory of the boat.

To determine this, we counted the number of calls in order to check if the boat had an influence on the CR. Fig.5 shows the result associated with the statistical tests. The CR means don't follow a normal distribution (Shapiro test, $\mathrm{p}$-value=0,0003), so the measure of the differences between the medians are calculated (Kruskal-Wallis Test, p-value=4.10e-05). After this the Dunn-Bonferonni test was done and the significant differences are showed in Fig.5.

During the first two periods (before the arrival of the boat), the density of calls is low. When the boat comes through BP (period $3)$, the density of calls started to increase until the boat leaves.

In fact, during period 4 (corresponding to the end of the passage of the boat) the CR is higher and significantly different from the first 2 periods. 
When the orcas turned around there were not as many calls (period 5). Then the density increased again when A108, I72 and 176 traveled together (period 6). This shows that the cruise ship had an influence on the type of calls as well as the CR.

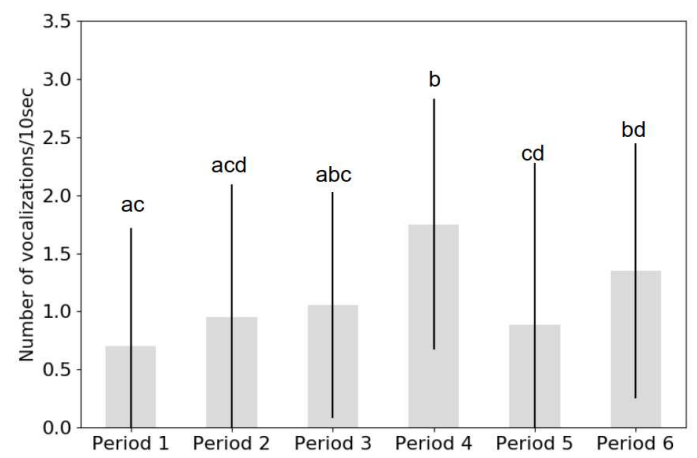

Figure 5. Graph of the CR of the $11^{\text {st }}$ of August for each period (1 to 6).

\section{Second observation ( $30^{\text {th }}$ of August)}

The second case study was on the $30^{\text {th }}$ of August in which the A42 and A25 matrilines of the A05 pod and the A54 matriline of the A01 pod came through BP together. Fig.6 illustrates the passage of this group. Each point represents a call placed in azimuth; the blue curve is the visual track of the group. Each visual observation (blue curve) matches the computed azimuth of each call. We thus validated the acoustic azimuth estimations.

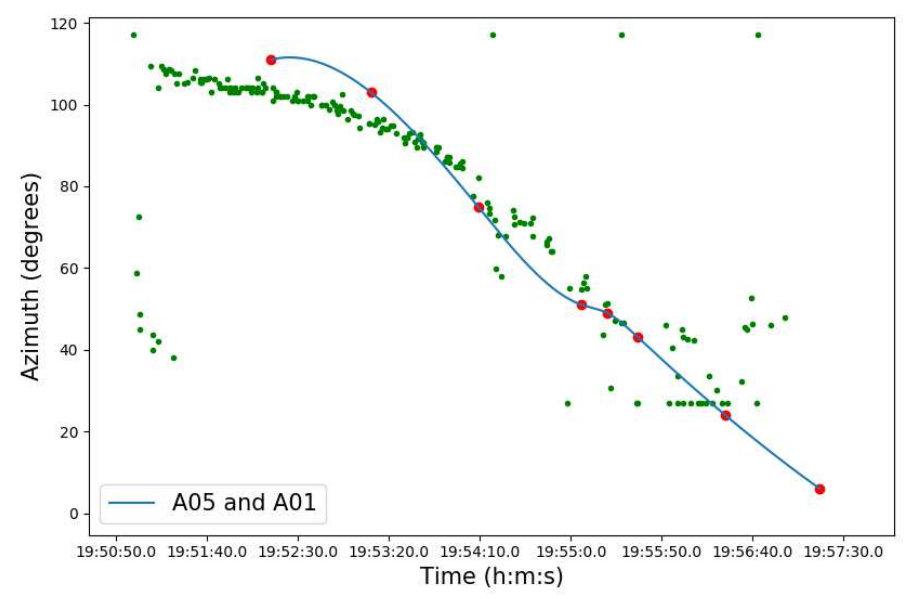

Figure 6. Graph of the passage of the $30^{t h}$ of August. Each green point represents a vocalization placed in azimuth. The blue curve is the interpolation of the A05 and A01 visual azimuth estimations.

Fig.7 represents the passage of the $30^{s t}$ of August in front of OL with examples of four pictures taken from OL. The visual observations confirmed that the group was very compact and traveled quickly (less than 6 minutes to traverse BP). 


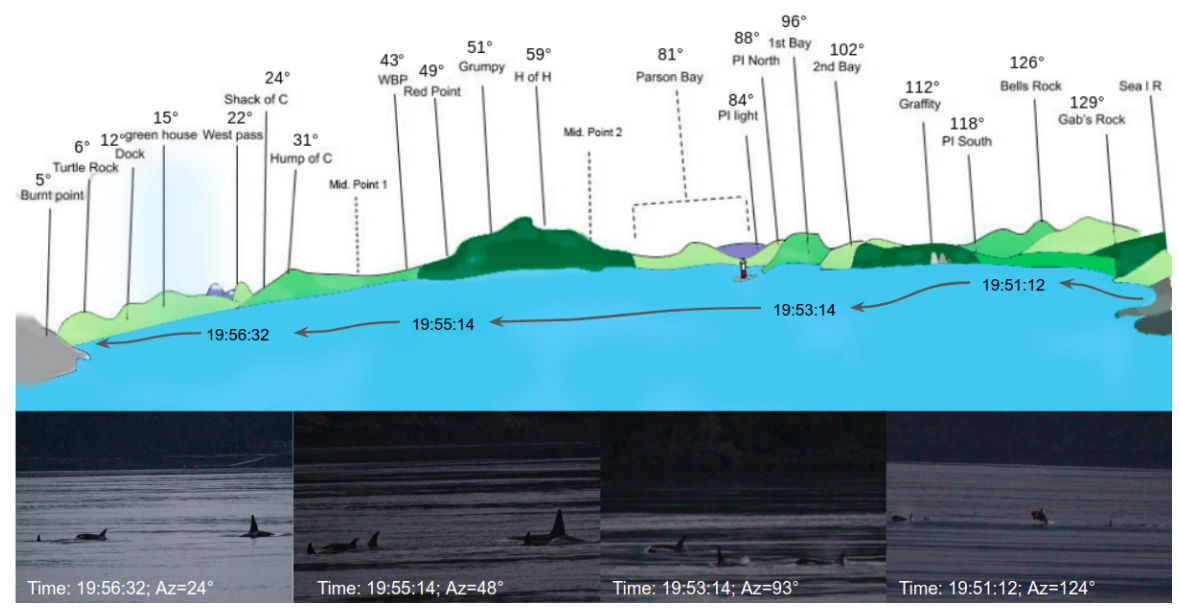

Figure 7. Graph of the passage of the $30^{\text {st }}$ of August. Top : representation of the view of BP from OL with the visual position of the group. Bottom : pictures taken from OL deck.

For each call, we extracted the type of call and plotted it according to the azimuth of the orcas (Fig.8).

The track is quite compact, and the group (A42s, A54s and A25s) travelled north very quickly. This group was composed of two different pods (A05 and A01). During this track, there were a lot of different calls which eventually subsided into the shared N03 call type. N03 could play a role in communication between these matrilines, because at the end of the track, individuals emitted only this call. It may indicate a change of behavior, i.e slowing down during travel.

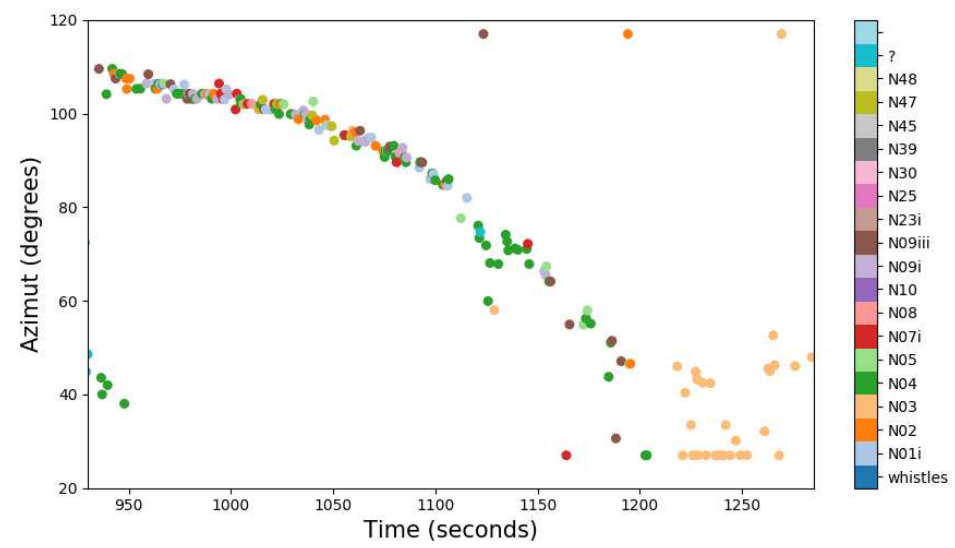

Figure 8. Graph of the passage of the $30^{\text {th }}$ of August. Each point represents a vocalization placed in azimuth. Each color represents a type of call.

Focusing on the track, we see that the A01s and A05s traveled together; attributed the calls to a pod following ${ }^{23}$. The Fig.9 shows the trajectory of A01s, A05s and their emitted calls. We divided the track into four parts and measured the CR in these periods. 


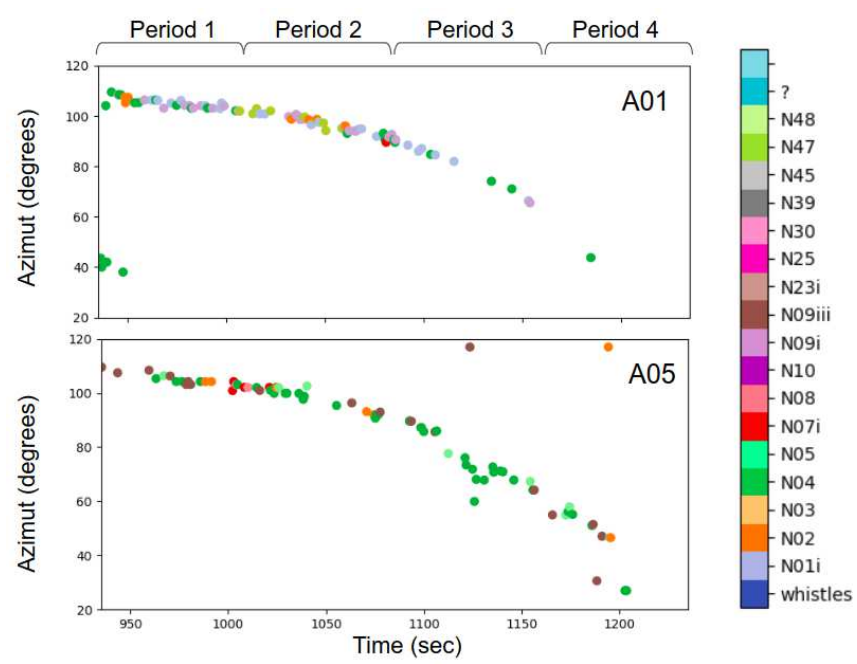

Figure 9. Graph of the passages of the $30^{\text {th }}$ of August. Each point represents a vocalization placed in azimuth. Each color represents a type of call. Top : trajectory of the A01s, Bottom : trajectory of the A05s.

We measured the Call Rate (CR) for the A01s and the A05s. The CR means don't follow a normal distribution (Shapiro test, $\mathrm{p}$-value $=0,0026$ ), so the differences between the medians were calculated (Kruskal-Wallis Test, $\mathrm{p}$-value $=1.10 \mathrm{e}-05$ ).

After this, Post-hoc testing was used to evaluate differences between each distribution pair (Dunn-Bonferroni tests) (Fig.10).

This graph shows significant differences between time periods and pods. During periods 1 and 2, the CR is higher for the A01 pod, then this is reversed for periods 3 and 4. During the last period (4), the CR for A01 pod is different from periods 1 and 2 .

When the density of calls of A01s increases, the CR of the A05s decreases. This result suggests that when these 2 pods travel together, A05s emit while A01s are silent, and vice versa. Hence, they do not overlap their calls, possibly maximizing their communication efficiency.

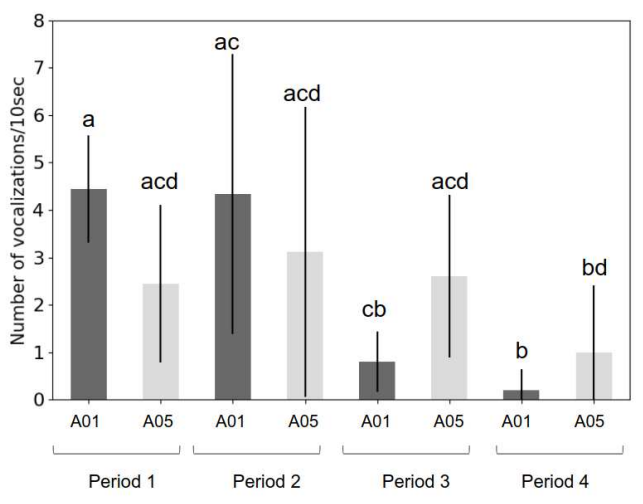

Figure 10. Graph of the CR of the $30^{s t}$ of August for each period and for the A01s (dark grey) and the A05s (light grey).

This case study showed us that when individuals were too close together it was difficult to identify matrilines or individuals by azimuth. But we were able to study the CR thanks to the acoustic identification of the different pods present.

\section{Third observation ( $31^{\text {st }}$ of August)}

We focused here on the $31^{s t}$ of August, when a large group group made up of I16s, I65s, I04s and I27s came through BP. The majority of the four matrilines traveled together; the male I76 (mat.I04), travelled by himself behind the large composite group, eventually joining them at the end of the track (Fig.11). Each point represents a vocalization placed by the estimated acoustic azimuth. The blue and orange curves are the interpolated visual azimuths of the male 176 and the large group, respectively. We see that it is possible to assign vocalizations either to the individual male or to the larger group because each acoustic position corresponds to a visual position. This result allowed us to isolate 24 calls from the male I76. 


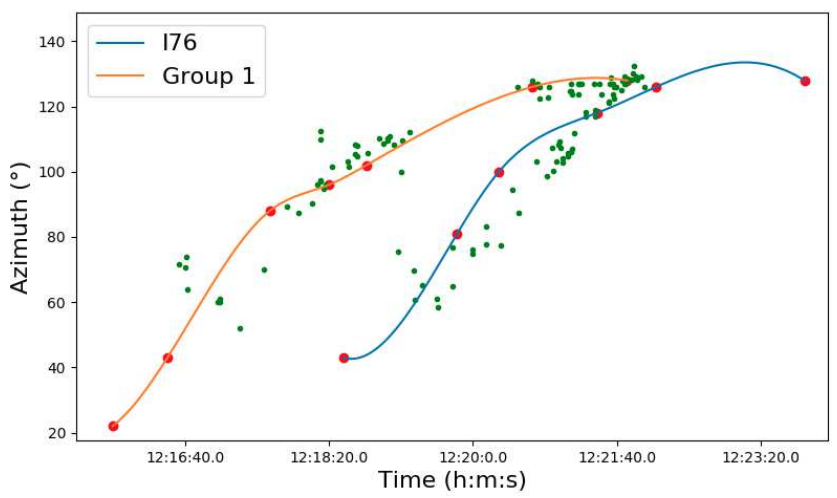

Figure 11. Graph of the passage of the $31^{s t}$ of August. Each green point represents a vocalization placed in azimuth. The blue curve is the interpolated position of the male I76 visually detected, the orange curve is the large group.

Fig.12 shows the visual trajectory photographed from OL and the representation of the trajectory in BP. The orange curve represents the large group and the white curve the male I76. Visual observations allowed us to validate our azimuthal positions from the acoustic antenna.

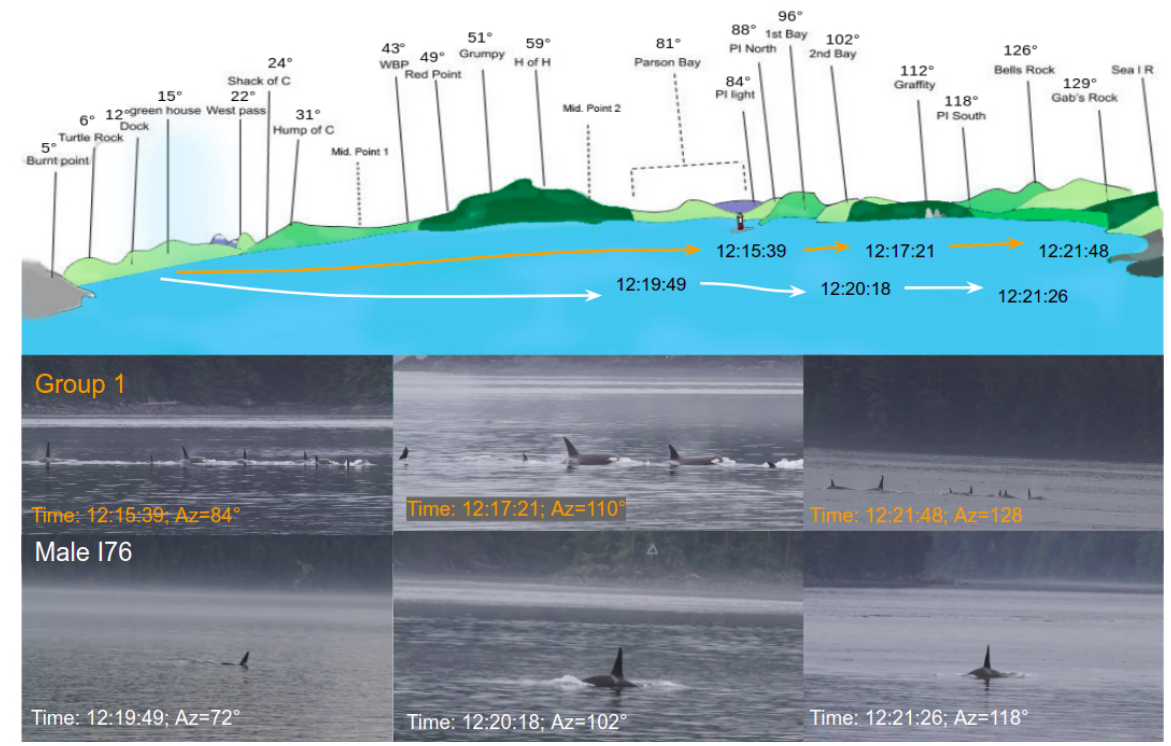

Figure 12. Graph of the passage of the $31^{\text {st }}$ of August. Top : representation of the view of BP from OL with the visual position of the group (orange) and the male I76 (white). Bottom : pictures taken from OL.

From the manual annotations, we made correlations between the azimuth and the type of calls for the large group and I76.

For these recordings, seven different calls were produced (Fig.13). Each point represents a call with its type in color. We see that the right track (I76) is composed of N48 (green), N25 (pink) and N23i (brown). Thus, we could assume that when the male was alone, he made a combination of two calls, N48 and N25, and when he was close enough to his group, he produced N23i calls to which his group answered with the same type. The large group produced all types of calls, including type N23i (brown) at the end of the track. We can see that the N23i type call is produced just before and after I76 merged with the larger group. 


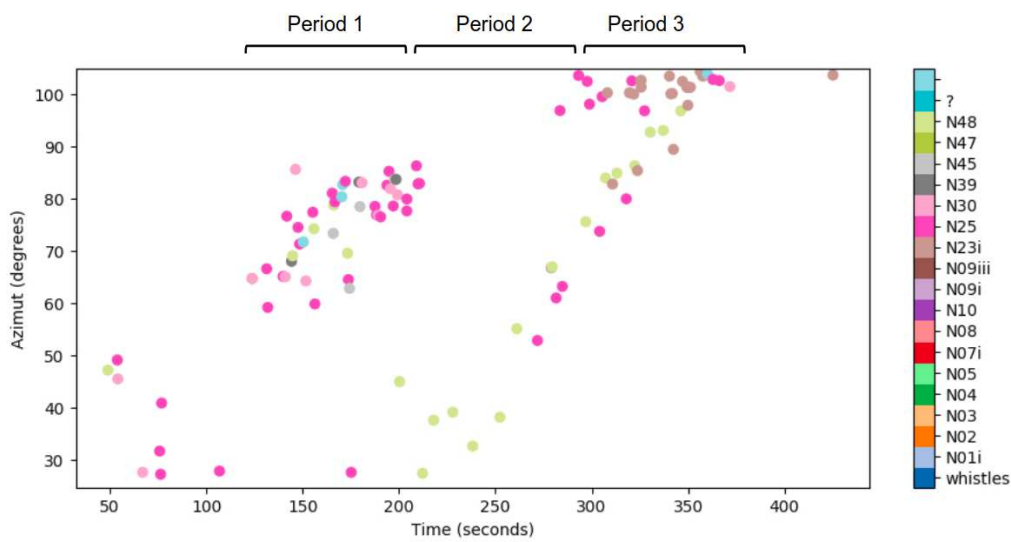

Figure 13. Graph of the passage of the $31^{\text {st }}$ of August. Each point represents a vocalization placed in azimuth. Each color represents a type of call. The most common types are N25, N48 and N23i. The track was split into three 80 s periods.

We measured the CR for the group and the male I76, in order to see if there were different periods of call exchanges. To do this, the number of vocalizations was counted every $10 \mathrm{~s}$ and its average was calculated for each period.

The means don't follow a normal distribution (Shapiro test, $\mathrm{p}$-value=0,0043), so the measure of the differences between the medians was calculated (Kruskal-Wallis Test, $\mathrm{p}$-value $=0,0022$ ).

So, $\mathrm{p}$-value $\leqslant \alpha$ : The differences between some of the medians are statistically significant. Post-hoc testing was used to evaluate differences between each distribution pair (Dunn-Bonferroni tests).

The result is showed in Fig 14. In the first period, only the large group was acoustically active compared to the male I76 (Post-hoc Bonferroni test, $\mathrm{p}<0.00006$ ). During the second and third periods, the group and $\mathrm{I76}$ had the same CR statistically. We observed that when the male started calling (period 2) the group did not call (compared to period 1). And during the third period (meeting) the CR of the group and I76 both increased.

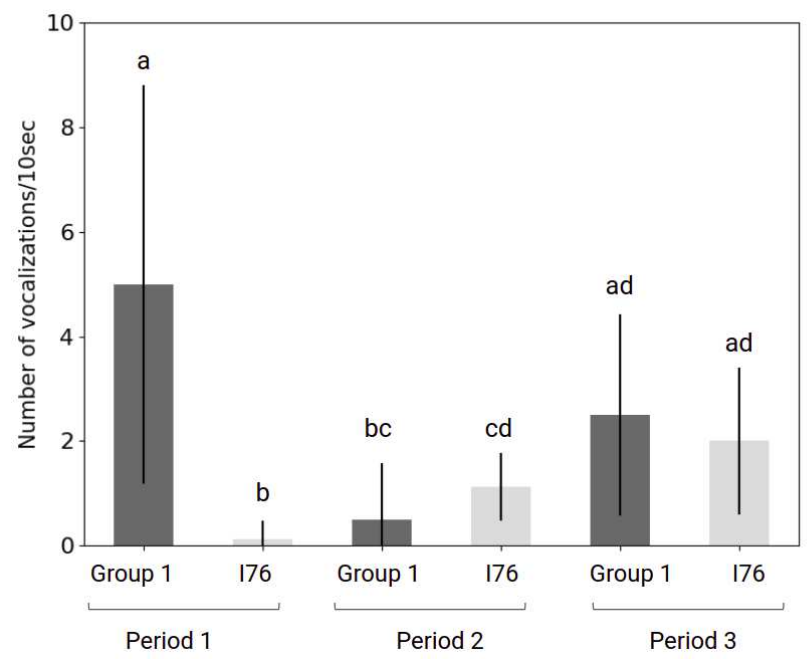

Figure 14. Graph of the CR of the $31^{\text {st }}$ of August for each period and for the group (dark grey) and the male I76 (light grey).

An animation is available for this case study at the address ${ }^{24}$.

\section{Discussion}

Orcas prefer group life, and in fact, it could have a variety of beneficial functions : localization of food, reproduction, socialization and traveling. In order to coordinate their activities, maintain group cohesion, or recognize members in the group, orcas need to communicate ${ }^{25}$.

Whistles have been shown to be important during socialization ${ }^{26}$. Echolocation clicks are used during hunting and to aid navigation. Discrete calls are often used during travel, hunting and socializing. The CR is different according to behavioral 
categories and particular events. In fact Grebner ${ }^{27}$ proved that orcas can initiate contact using discrete pulsed calls during pre-joining events.

Our results show that we can track individual calls of wild orcas in their natural habitat in order to demonstrate Call Rate (CR) modulations according to various parameters including, the presence of boats, and the relative position of an individual versus a group of whales. To accomplish this we analyzed three different natural scenes or observations.

The first observation presented a cruise ship travelling through the A05s and A01s while they were grouped together. Before the boat arrived, they moved at a slow speed and produced a lot of different calls (Fig.2). Once the boat arrived on the scene, the orcas followed it and produced specific types of calls (N04 and N47). After the boat exited BP and left the orcas behind, many N03 calls were emitted and some of the orcas turned around. The CR increased significantly at the end of the passage of the boat and when the boat moved away, whereas the CR was stable before the arrival of the boat.

Anthropogenic noise has an influence on orca emissions. Holt ${ }^{18}$ has proven that orcas increase their call amplitude in response to vessel noise. Our study complements this observation, showing that orcas change both their trajectory and CR when a large vessel is present.

In fact, both large (e.g. cruise ship or tanker) and small vessels are very noisy and produce noise in the 10-1000 Hz range. The propagation in water is important, resulting in frequencies that could span more than $5 \mathrm{kHz}$. There is an overlap between the vocalizations emitted for communication and anthropogenic noise. Cetaceans may have different options to adapt : they can maintain the communication by increasing the call amplitude (Lombard effect) ${ }^{28}$, they can increase the duration of the signal ${ }^{14}$, or they can change the type of their calls or their CR to avoid the overlap with the boat noise ${ }^{29,30}$.

Here, it seems we have an example of the last option.

In the second observation ( $30^{\text {th }}$ of August), one big group passed in front of OL, composed of A05 pod (mat. A42 and A25), and the A01 pod (mat. A54).

The tracks of these groups were interesting : two pods travelled together and exchanged a lot of calls for several minutes and at the end of the trajectory a lot of N03 calls were emitted. $\mathrm{In}^{4}$ it was realised that N03 calls are produced after periods of social activity or high-arousal contexts like active foraging. Moreover, the lower source level of the N03 call makes this call more suitable during close range vocal exchanges ${ }^{31}$.

We found that pods emit calls in turns, alternating low and high CR when travelling together and the track is split in different parts in time (Fig.9), or when there are particular combinations of calls. Our study shows that individuals from different pods (A05 and A01) can communicate and travel together. Resident orcas are organized in a fission-fusion society that is flexible in time and space.

We propose two hypotheses : first, when more than one pod travels closely and one pod begins communicating, individuals from the other pod will try to be silent in order to facilitate the communication between individuals of the first pod.

Second, and following ${ }^{15}$, we can assume the existence of a veritable interaction between these two pods. For example, when the first pod vocalizes, then a $2^{\text {nd }}$ pod will respond to them (and vice-versa).

According to the second hypothesis, the type of call provides particular information to the original signaler : the combination of particular calls from A05 depends on the emission of particular A01 calls (Fig.9).

In the third observation, on the $31^{\text {th }}$ of August, a big group made up of the I16, I65, I04 and I27 matrilines came through BP. Behind them was a lone male orca, I76 (mat. I04). This scene is noteworthy because the male traveled far behind his group and made specific types of calls. When he was far from his group he made a combination of N25 and N48 calls, but once he approached his group, he vocalized with the N23i call type and his group answered with the same type of call. We conclude that any modulations of call types could improve the ability of orcas to give information on their relative positions and help to localize each other.

Furthermore, there may be a greater need for redundancy in communication signals in large groups as is typical of common dolphins, requiring faster signal production to facilitate inter-individual or group cohesion. Our results have complemented the study of ${ }^{27}$ who highlighted the production of calls before the meeting of two groups. Orcas could use particular types of calls in order to improve good propagation in the water and facilitate the reception of the signal.

When somewhat isolated, the male made a particular type of call and modulated his CR (Fig.11). As he got closer to his group he increased his CR (period 3) and emitted other call types in the direction of his group ${ }^{32}$ presumably in order to facilitate communication. Additionally, the group decreased its CR. Such changes in the CR may occur when individuals are separated from their group. A similar pattern is shown for other species : Bottlenose dolphins ${ }^{33}$, Spider monkeys ${ }^{34}$ and Chimpanzees ${ }^{35}$. To sum up, this study of nearly one hundred calls suggests that intra-group acoustic communication in orcas may depend on the :

- distance between the group and the individual,

- acoustic emissions of another pod,

- presence of a boat, 
- physical behavior of individuals (surfing behind a boat).

Our shore based study looked at orca communication in the wild at a unique level of precision.

Other shore based fixed hydrophone systems enabled the detection and tracking of orcas from the same population ${ }^{36-38}$ but did not succeed in associating individuals with calls, or determine precise individual pattern variations of communication between groups.

This work does not pretend to provide all explanations for the type of calls, but rather the CR. Results offer evidence that this low cost and easy to deploy protocol, brings to light relevant features of CR of these complex communication systems.

\section{Material and Methods}

Over the past 35 years, the non-governmental organization OrcaLab (OL), has developed and maintained a multi-hydrophone recording system around Hanson Island (northern Vancouver Island), Canada to study whales. Throughout the year, OL consistently observes and records orca behaviors and vocalizations. To increase the knowledge surrounding orca communication, our team designed a small aperture four-hydrophone array at a high sampling rate ( $4 * 192 \mathrm{kHz} 16$ bits). One goal was to record highly defined acoustic data from orcas passing in front of OL and connect these data to visual observations made from land at OL. The goal was to estimate the Time Delays of Arrival (TDoA) of the calls, thus their azimuth by a simple linear solver, and to assess a multi-modal label for each call : the type of call, azimuth and identity of the emitting orca.

Finally, we studied the Call Rate (CR) of the emitting individual/matriline.

\section{Acoustic data acquisition}

The four-hydrophone array is a tetrahedral portable antenna of $12 \mathrm{~kg}$ (Fig.15) that uses four SQ26-01 hydrophones with a high frequency sensibility between $10 \mathrm{~Hz}$ to $50 \mathrm{kHz}$. The flat frequency response of the hydrophones is available in supplemental material. The maximum aperture of this array is $77 \mathrm{~cm}$.

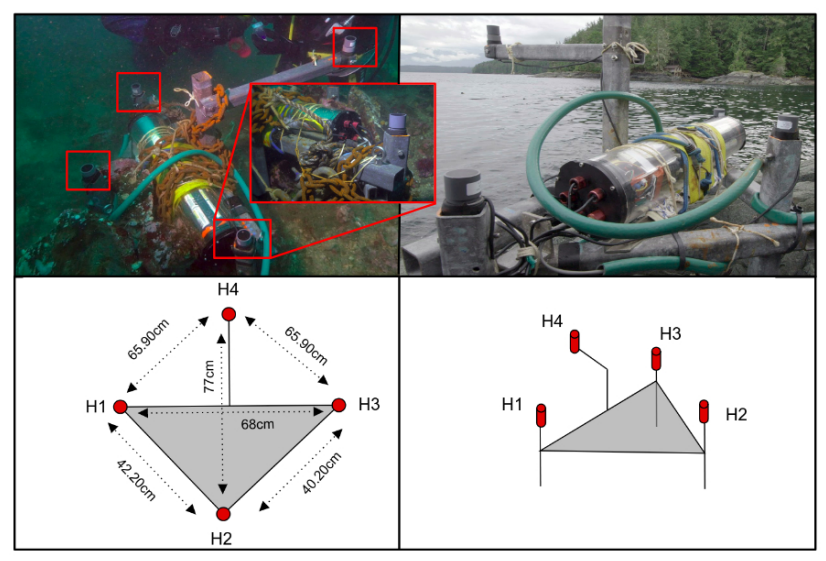

Figure 15. Top : the four-hydrophone antenna, with the $12 \mathrm{~V}$ (green) cable connected into OL. Bottom : diagram of the antenna and its inter-hydrophone distances.

It includes a novel low-power high-resolution autonomous sound card, called JASON ${ }^{39}$ (Fig.16), and the recording capability of up to 5 uncompressed channels at a sampling rate of up to $2 \mathrm{MHz}, 16$ bits. It can be accompanied by a low power acoustic trigger $^{40}$, but this was not necessary at OL because JASON was switched ON/OFF manually from OL when orcas were seen.

In this study we recorded at a $192 \mathrm{kHz}$ sampling rate on four channels (Supplementary material : A recordings samples are available from the $11^{\text {th }}, 30^{\text {th }}, 31^{\text {th }}$ of August, 4 channels, $192 \mathrm{kHz} \mathrm{SR}$ ).

We over-sampled the bandwidth of the hydrophones $(50 \mathrm{kHz})$ to increase the precision of the estimated angle of arrival and to increase the efficiency of separating the calls individual by individual. The antenna was placed at a depth of $23 \mathrm{~m}$ pointing East, which was determined using a compass. Due to its weight and an additional $10 \mathrm{~kg}$ of ballast there was no concern regarding its stability, even during strong currents (> $1 \mathrm{~m} / \mathrm{s})$.

The device was powered from the shore through $75 \mathrm{~m}$ of $12 \mathrm{~V}$ cable connected to OL. The ability to power the device from land allowed us to limit our recording to when orcas passed in front of OL. Later, we could correlate the acoustic data with visual observations from OL. The antenna was taken out of the water when the 512 Go uSD storage was full, when we reached 
74 hours of recording, or earlier in order to avoid obstacles, such as sea urchins, from settling on the antenna and interfering with the recording.

The recording sessions took place from the $20^{\text {th }}$ of July to the $1^{\text {st }}$ of August, the $4^{\text {th }}$ of August to the $22 n d$ of August, and the $23^{\text {rd }}$ of August to the $15^{\text {th }}$ of September 2019. In total, we recorded 42 vocal passages that included multiple orcas.

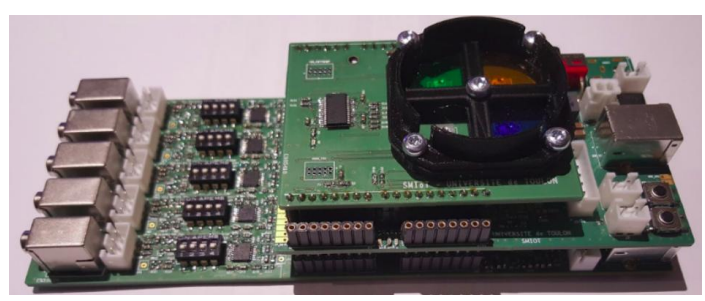

Figure 16. The JASON sound card $5 \times 2 \mathrm{MHz}$ sampling rate at 16 bits resolution, placed into the submarine tube (the luxmeter was not used here).

\section{Visual data acquisition}

When orcas were spotted in BP located in front of OL. The antenna was powered on by a simple on/off button from OL. It was turned off when the orcas were no longer visible (Fig.17). During each passing of the orcas, 5 observers at OL worked together to identify the groups and individuals present. This task was always carried out by the same people :

- Person 1 (H.S.) : Real-time identification of groups and individuals (when possible) with a dedicated 'Big Eyes' binocular ${ }^{41}$,

- Person 2 (P.S.) : Using spotting scope to help H.S. for identifications (Bushnell Spacemaster 78-1800 with, zoom 15x45),

- Person 3 (M.P.) : Recording of Helena's real time comments and azimuths of each individual/matriline associated with the visual reference points in BP. Each site was assigned a precise azimuth with a compass (eg. "Green House" $=15$ degrees). At every surfacing the azimuth was recorded,

- Person 4 (A.Doohan) : Recording of "Big Eyes" positions and photographing individuals,

- Person 5 (E.Vierling) : photographing individuals.

If several groups were present at different azimuths, we used a second "Big Eyes" to make the identifications. Once the passage was finished, the whole team confirmed the presence of the individuals using the photographs and the photoidentification catalogue ${ }^{42}$. 


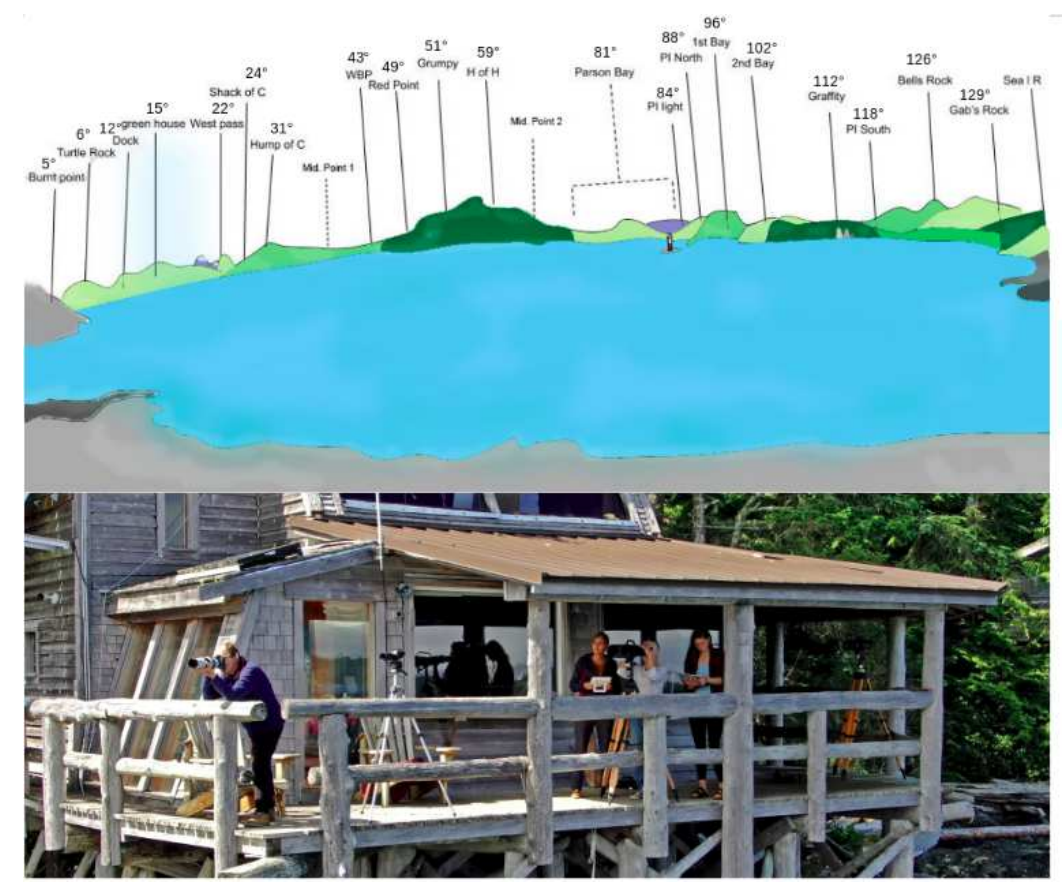

Figure 17. (Top) Representation of the view of BP from OL. Each site was assigned a precise azimuth using a compass (ex : "Pi Light" = 84 degrees.). (Bottom) Picture of the visual protocol.

\section{Annotation of the recordings}

Manual annotations of the vocalizations were conducted with Audacity to provide waveform and spectrogram visualization ${ }^{43}$. Three recordings were precisely annotated in which calls were identified and correlated (Fig.18).

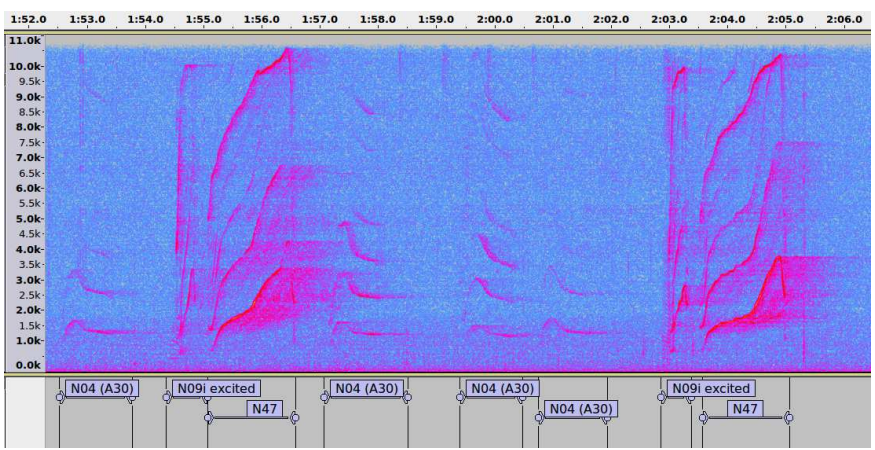

Figure 18. Example of annotation of $13 \mathrm{~s}$ of recordings (Audacity). Hanning window size : 1024.

\section{Azimuth estimation of the orca calls using the hydrophone array}

As described in the previous section, each vocalization was detected and annotated. From the four channels we computed the three independent Time Delay of Arrival (TDoA) by inter-correlations : $\tau_{21}, \tau_{31}, \tau_{41}$. The high sampling rate $(192 \mathrm{kHz})$ allowed us to measure precise TDoAs. At relatively far range, the sinus of the angle of arrival is equal to the angle of arrival. Then we estimated the azimuths with a linear system as presented $\mathrm{in}^{44}$. Range estimation of the calls was possible in certain conditions $^{45,46}$, but due to surface acoustic effects, we did not estimate the range of orcas in our protocol. However, we show that azimuth estimations are accurate for this study. Then, we joined the azimuth estimates of each call to every identified orca using the visual positions noted by OL observers. We associated individuals or groups of orca with a vocalization track by using a photo-identification catalogue ${ }^{42}$. When orcas were very close to each other, it was difficult to associate a call with an individual, but in some cases we were able to associate vocalizations with a specific male. 


\section{Calculation of the Call Rate and statistical tests}

Once the individual/matriline were identified we used a quantitative variable : the Call Rate (CR) by counting the number of vocalizations every $10 \mathrm{~s}$ for each individual/matriline. Thus, we divided the tracks into different periods $(80$, or $5 * 80=400 \mathrm{~s})$, and we estimated the mean and variance of the CR over the non overlapping $10 \mathrm{~s}$ intervals in each period. Then we performed statistical tests between periods and individual/matriline. Accuracy was not normally distributed as assessed by Shapiro-Wilk's test ( $\mathrm{p}$-value $<0.001)$. Since our data did not follow a normal distribution, we performed a Kruskal-Wallis Test ${ }^{47}$ followed by a post Hoc test of Dunn-Bonferroni ${ }^{48}$ (Fig.19).

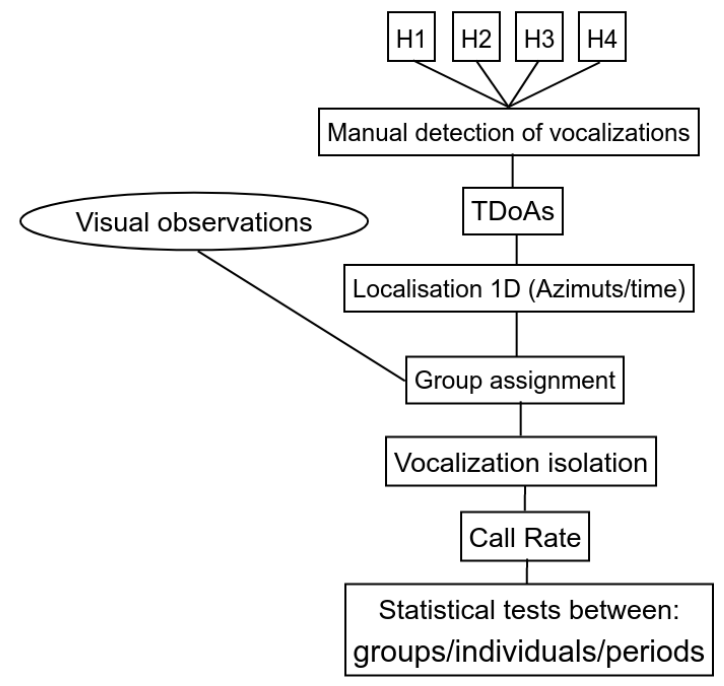

Figure 19. Synopsis of the protocol.

\section{References}

1. National Research Council and others, Nat. Acad. Press. Ocean noise and marine mammals (2003).

2. Burham, R., Palm, R., Duffus, D., Mouy, X. \& Riera, A. The combined use of visual and acoustic data collection techniques for winter killer whale (Orcinus orca) observations. Glob. ecology conservation 8, 24-30 (2016).

3. Ford, J. K. Vocal traditions among resident killer whales (Orcinus orca) in coastal waters of British Columbia. Can. journal zoology 69, 1454-1483 (1991).

4. Ford, J. K. Acoustic behaviour of resident killer whales (Orcinus orca) off vancouver island, british columbia. Can. J. Zool. 67, 727-745 (1989).

5. Filatova, O., Guzeev, M., Fedutin, I., Burdin, A. \& Hoyt, E. Dependence of killer whale (Orcinus orca) acoustic signals on the type of activity and social context. Biol. Bull. 40, 790-796 (2013).

6. Bain, D. Acoustic behavior of Orcinus: Sequences, periodicity, behavioral correlates and an automated technique for call classification. Behav. biology killer whales 335-371 (1986).

7. Filatova, O., Burdin, A. \& Hoyt, E. Horizontal transmission of vocal traditions in killer whale (Orcinus orca) dialects. Biol. bulletin 37, 965-971 (2010).

8. Bigg, M., Olesiuk, P., Ellis, G. M., Ford, J. \& Balcomb, K. C. Social organization and genealogy of resident killer whales (Orcinus orca) in the coastal waters of British Columbia and Washington state. R. Int. Whal. Comm. 12, 383-405 (1990).

9. Hoelzel, A. R. \& Dover, G. A. Genetic differentiation between sympatric killer whale populations. Heredity 66, 191-195 (1991).

10. Ford, J. K. et al. Dietary specialization in two sympatric populations of killer whales (Orcinus orca) in coastal British Columbia and adjacent waters. Can. J. Zool. 76, 1456-1471 (1998).

11. Jones, I. M. A northeast pacific offshore killer whale (Orcinus orca) feeding on a pacific halibut (Hippoglossus stenolepis). Mar. mammal science 22, 198-200 (2006). 
12. Ford, J. K. Killer whale: (Orcinus orca). In Encyclopedia of marine mammals, Elsevier, 650-657 (2009).

13. Miller, P. J., Shapiro, A., Tyack, P. \& Solow, A. Call-type matching in vocal exchanges of free-ranging resident killer whales, (Orcinus orca). Animal Behav. 67, 1099-1107 (2004).

14. Foote, A. D., Osborne, R. W. \& Hoelzel, A. R. Whale-call response to masking boat noise. Nature 428, 910-910 (2004).

15. Krebs, J. R., Ashcroft, R. \& Van Orsdol, K. Song matching in the great tit Parus major L. Animal Behav. 29, 918-923 (1981).

16. Janik, V. M. Whistle matching in wild bottlenose dolphins (Tursiops t.). Science 289, 1355-1357 (2000).

17. Miller, P. J. \& Tyack, P. L. A small towed beamforming array to identify vocalizing resident killer whales (Orcinus orca) concurrent with focal behavioral observations. Deep. Sea Res. Part II: Top. Stud. Oceanogr. 45, 1389-1405 (1998).

18. Holt, M. M., Noren, D. P., Veirs, V., Emmons, C. K. \& Veirs, S. Speaking up: Killer whales (Orcinus orca) increase their call amplitude in response to vessel noise. The J. Acoust. Soc. Am. 125, EL27-EL32 (2009).

19. Luís, A. R., Couchinho, M. N. \& dos Santos, M. E. Changes in the acoustic behavior of resident Bottlenose dolphins near operating vessels. Mar. Mammal Sci. 30, 1417-1426 (2014).

20. Quick, N. J. \& Janik, V. M. Whistle rates of wild Bottlenose dolphins (Tursiops truncatus): Influences of group size and behavior. J. Comp. Psychol. 122, 305 (2008).

21. Reers, H. \& Jacot, A. The effect of hunger on the acoustic individuality in begging calls of a colonially breeding weaver bird. BMC ecology 11, 3 (2011).

22. Leonard, M. L., Horn, A. G. \& Parks, E. The role of posturing and calling in the begging display of nestling birds. Behav. Ecol. Sociobiol. 54, 188-193 (2003).

23. Ford, J. K. et al. A catalogue of underwater calls produced by killer whales (Orcinus orca) in British Columbia (Department of Fisheries and Oceans, Fisheries Research Branch, Canada, 1987).

24. Rose, Y. Animation of the $30^{\text {th }}$ of august. http://sabiod.univ-tln.fr/media/30_08_Orcalab.mp4 (2020).

25. Grebner, D. M. et al. Localization of killer whale mother, juvenile, and calf vocalizations. The J. Acoust. Soc. Am. 124, 2507-2507 (2008).

26. Thomsen, F., Franck, D. \& Ford, J. K. On the communicative significance of whistles in wild killer whales (Orcinus orca). Naturwissenschaften 89, 404-407 (2002).

27. Grebner, D. M. Individual killer whale vocal variation during intra-group behavioral dynamics. Phd Thesis, Pennsylvania State Univ. (2009).

28. Noad, M., Dunlop, R. \& Cato, D. The lombard effect in Humpback whales. J. Acoust. Soc. Am. 131, 3456-3456 (2012).

29. Castellote, M., Clark, C. W. \& Lammers, M. O. Acoustic and behavioural changes by fin whales (Balaenoptera physalus) in response to shipping and airgun noise. Biol. Conserv. 147, 115-122 (2012).

30. Poupard, M., de Montgolfier, B. \& Glotin, H. Ethoacoustic by bayesian non parametric and stochastic neighbor embedding to forecast anthropic pressure on dolphins. In IEEE OCEANS, 1-5 (2019).

31. Miller, P. J. Diversity in sound pressure levels and estimated active space of resident killer whale vocalizations. J. Comp. Physiol. A 192, 449 (2006).

32. Miller, P. J. Mixed-directionality of killer whale stereotyped calls: A direction of movement cue? Behav. Ecol. Sociobiol. 52, 262-270 (2002).

33. Cook, M. L., Sayigh, L. S., Blum, J. E. \& Wells, R. S. Signature-whistle production in undisturbed free-ranging Bottlenose dolphins (Tursiops truncatus). Proc. Royal Soc. London. Ser. B: Biol. Sci. 271, 1043-1049 (2004).

34. Ramos-Fernández, G. Vocal communication in a fission-fusion society: do spider monkeys stay in touch with close associates? Int. J. Primatol. 26, 1077-1092 (2005).

35. Mitani, J. C. \& Nishida, T. Contexts and social correlates of long-distance calling by male chimpanzees. Animal Behav. 45, 735-746 (1993).

36. Poupard, M. et al. Deep learning for ethoacoustics of orcas on three years pentaphonic continuous recording at OrcaLab revealing tide, moon and diel effects. In IEEE OCEANS, 1-7 (2019).

37. Bergler, C. et al. Orca-spot: An automatic killer whale sound detection toolkit using deep learning. Sci. reports 9, 1-17 (2019). 
38. Best, P. et al. Deep learning and domain transfer for orca vocalization detection. In Proc. Int. J. Conf on Neural Net $(I J C N N)(2020)$.

39. Barchasz, V., Gies, V., Marzetti, S. \& Glotin, H. A novel low-power high speed accurate and precise DAQ with embedded artificial intelligence for long term biodiversity survey. In Proc. Acustica Symp. (2020).

40. Fourniol, M. et al. Low-power wake-up system based on frequency analysis for environmental internet of things. In IEEE Int. Conf. on Mech. and Embedded Sys. and App. (MESA), 1-6 (2018).

41. Product, R.-C. B. E. Big eyes ${ }^{\mathrm{TM}}$ binoculars - product repair. Website: http://www.bigeyes.ca/f_03_specifications_binoculars. htm (2014).

42. Towers, J. Photo-identification catalogue and status of the northern resident killer whale population in 2014 (Fisheries and Oceans Canada, 2015).

43. Audacity-Team. Audacity: free audio editor and recorder. Website: https://www.audacityteam.org/ (1999).

44. Bénard-Caudal, F., Giraudet, P. \& Glotin, H. Whale 3D monitoring using astrophysic nemo onde two meters wide platform with state optimal filtering by rao-blackwell monte carlo data association. Appl. acoustics 71, 994-999 (2010).

45. Glotin, H., Caudal, F. \& Giraudet, P. Whale cocktail party: real-time multiple tracking and signal analyses. Can. acoustics 36, 139-145 (2008).

46. Poupard, M. et al. Real-time passive acoustic 3D tracking of deep diving cetacean by small non-uniform mobile surface antenna. In Int. Conf. on Acoustics, Speech and Signal Proc. (IEEE ICASSP), 8251-8255 (2019).

47. Breslow, N. A generalized kruskal-wallis test for comparing k samples subject to unequal patterns of censorship. Biometrika 57, 579-594 (1970).

48. Dunn, O. J. Multiple comparisons using rank sums. Technometrics 6, 241-252 (1964).

49. CRT. Cetacean research technology. Website: https://www.cetaceanresearch.com/hydrophones/sq26-01-hydrophone/index. html (1994).

\section{Acknowledgements}

We thank OrcaLab and its volunteers for welcoming M. Poupard and H. Glotin during the summer of 2019 to set up this protocol. We thank M. Hockin-Bennett for her indispensable help on the dives. We thank E. Vierling and A. Doohan for their essential support. We thank T. Lengagne, T. Soriano for discussions. We thank SMIoT Scientific Instrumentation (V. Gies, V. Barchasz), and INPS of UTLN for the development of the JASON DAQ project.

We thank BIOSONG SAS for the co-funding of M. Poupard's PhD. This research is partly funded by : FUI 22 Abyssound, ANR-18-CE40-0014 SMILES, ANR-17-MRS5-0023 NanoSpike, ADSIL ANR-20-CHIA-0014-01 National H.G. Chair in AI for bioacoustics, MARITTIMO European FEDER GIAS, Région SUD SEAMED, IUF, and MI CNRS MASTODONS sabiod.org.

\section{Author contributions statement}

All authors conceived the experiment.

H.G. and M.P. defined the protocol.

M.P. and H.G. have built and prepared the antenna.

All authors participated with the installation the antenna.

H.S., P.S. and M.P. participated in the visual data acquisition.

H.S. annotated the recordings with Audacity.

M.P. and H.G. computed the detection and the azimuth of the vocalisations.

M.P., H.G. and H.S. wrote the manuscript and produced the results.

All authors discussed the results and reviewed the manuscript.

\section{Additional information}

The recordings samples are available from the $11^{\text {th }} 30^{\text {th }}, 31^{\text {th }}$ of August, 4 channels, $192 \mathrm{kHz}$ SR 16 bits (under copyright of the authors) at the address: http://sabiod.univ-tln.fr/pub/orcas $R /$

The frequency response of the hydrophone SQ26 is in Fig.20. 


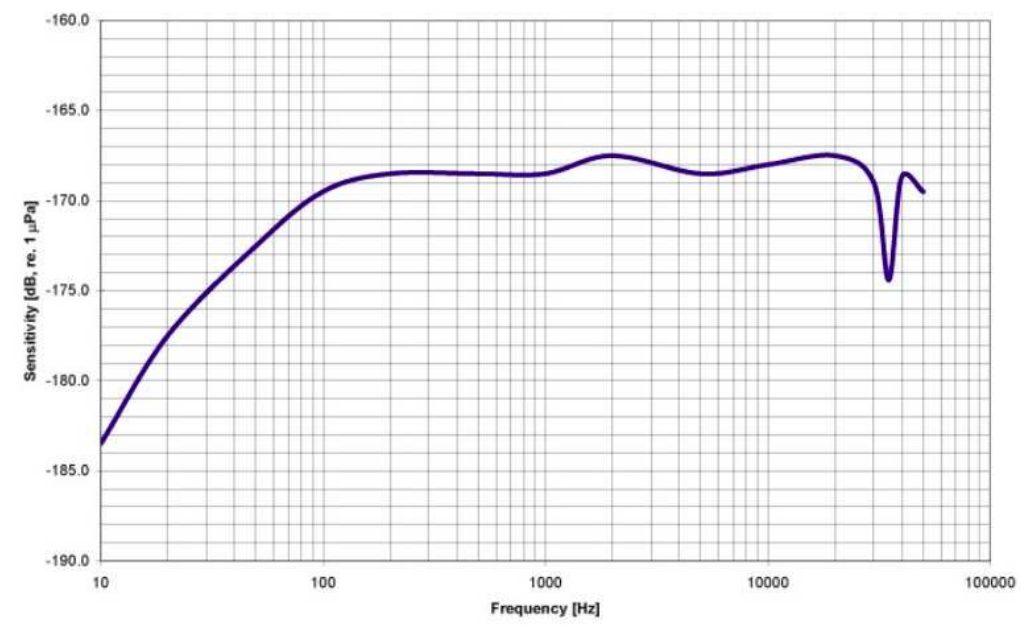

Figure 20. SQ26-07 Frequency response ${ }^{49}$ 
Figures

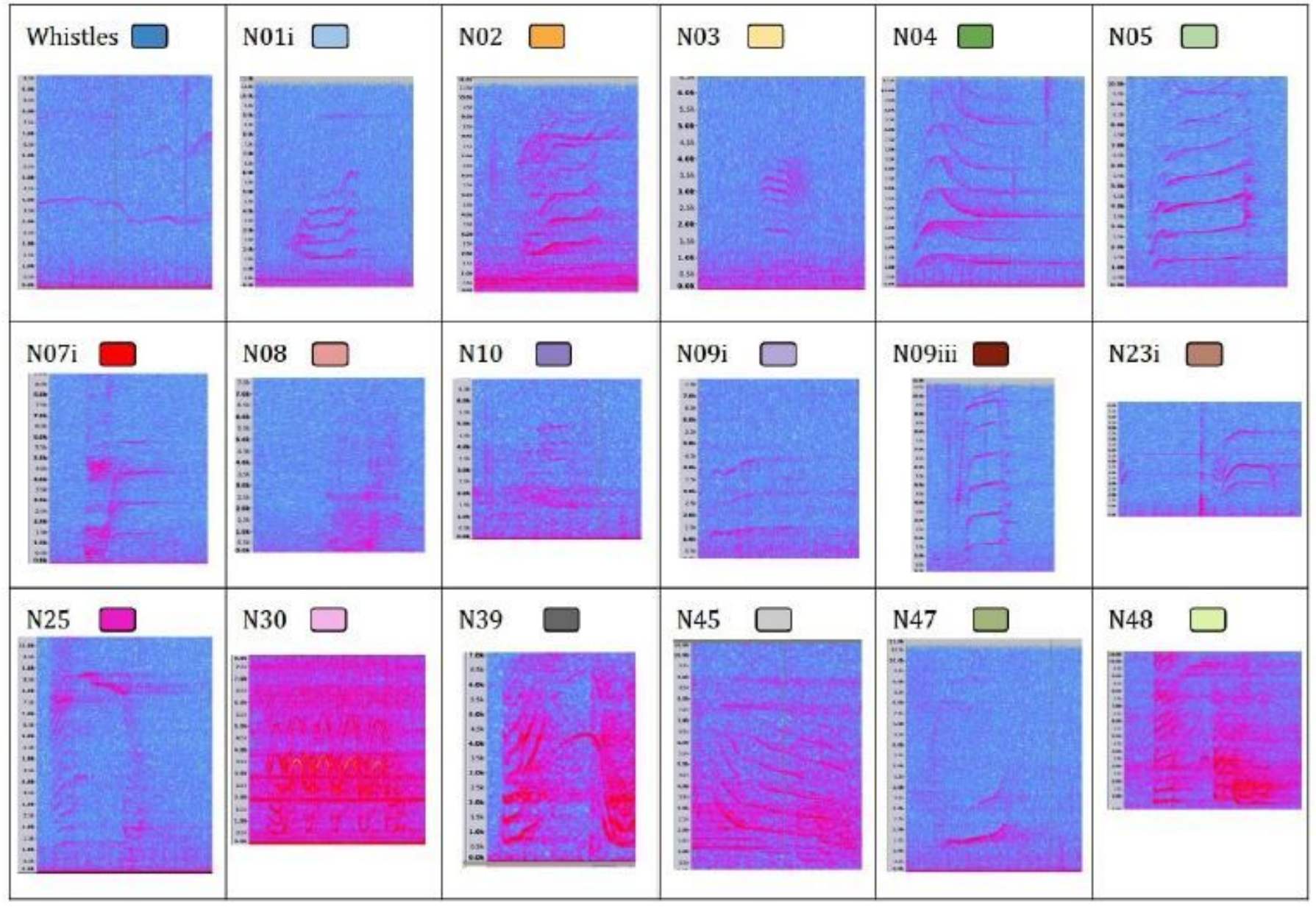

Figure 1

List of the calls annotated in each experiment. 


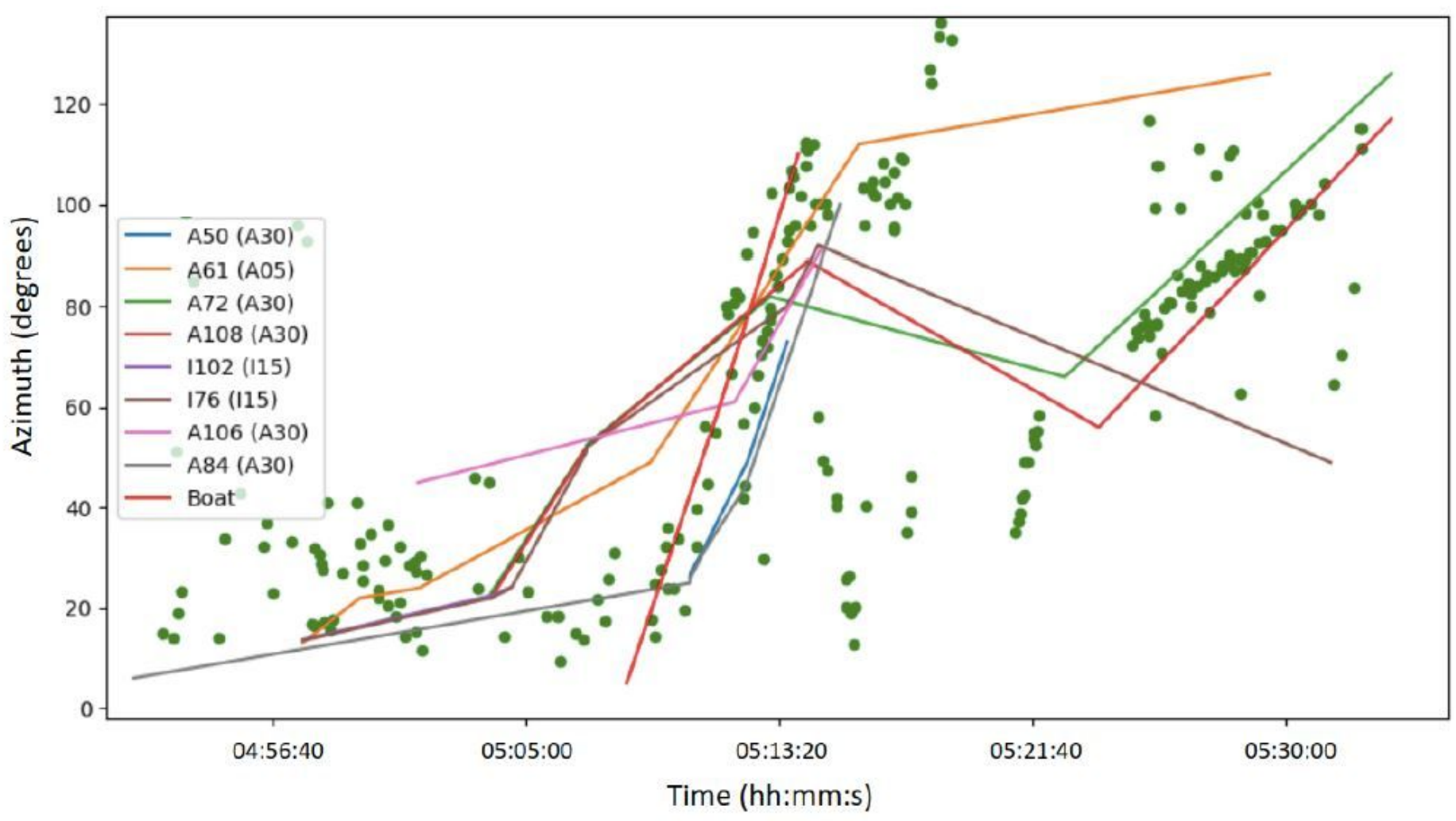

Figure 2

Graph of the passage of the 11th of August. Each green point represents a vocalization placed in azimuth. The different curves are the positions of each individual visually detected.

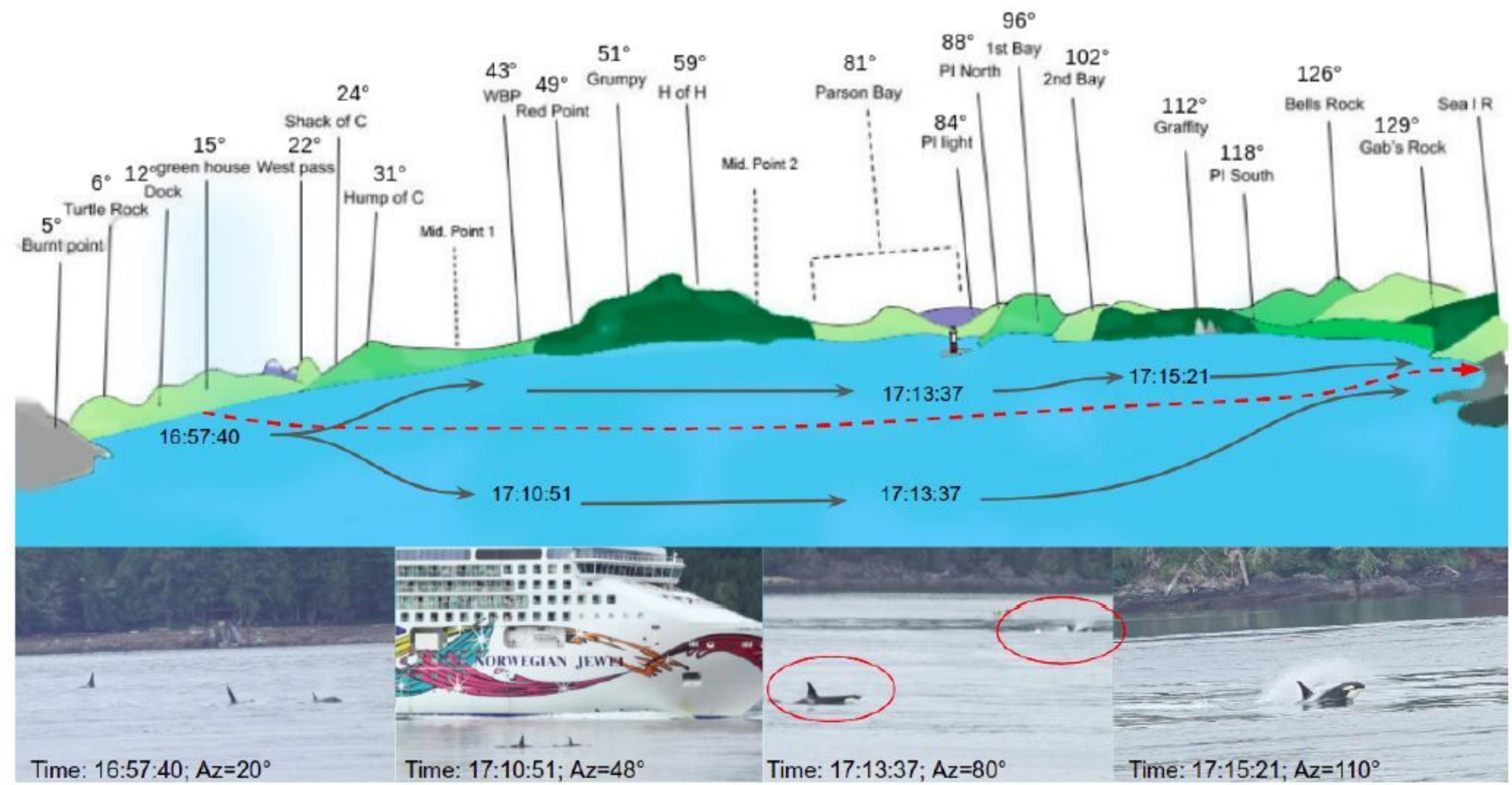


Figure 3

Graph of the passage of the 11th of August. Top : representation of the view of BP from OL with the visual position of the group. The red line is the boat trajectory. Bottom : pictures taken from OL deck.

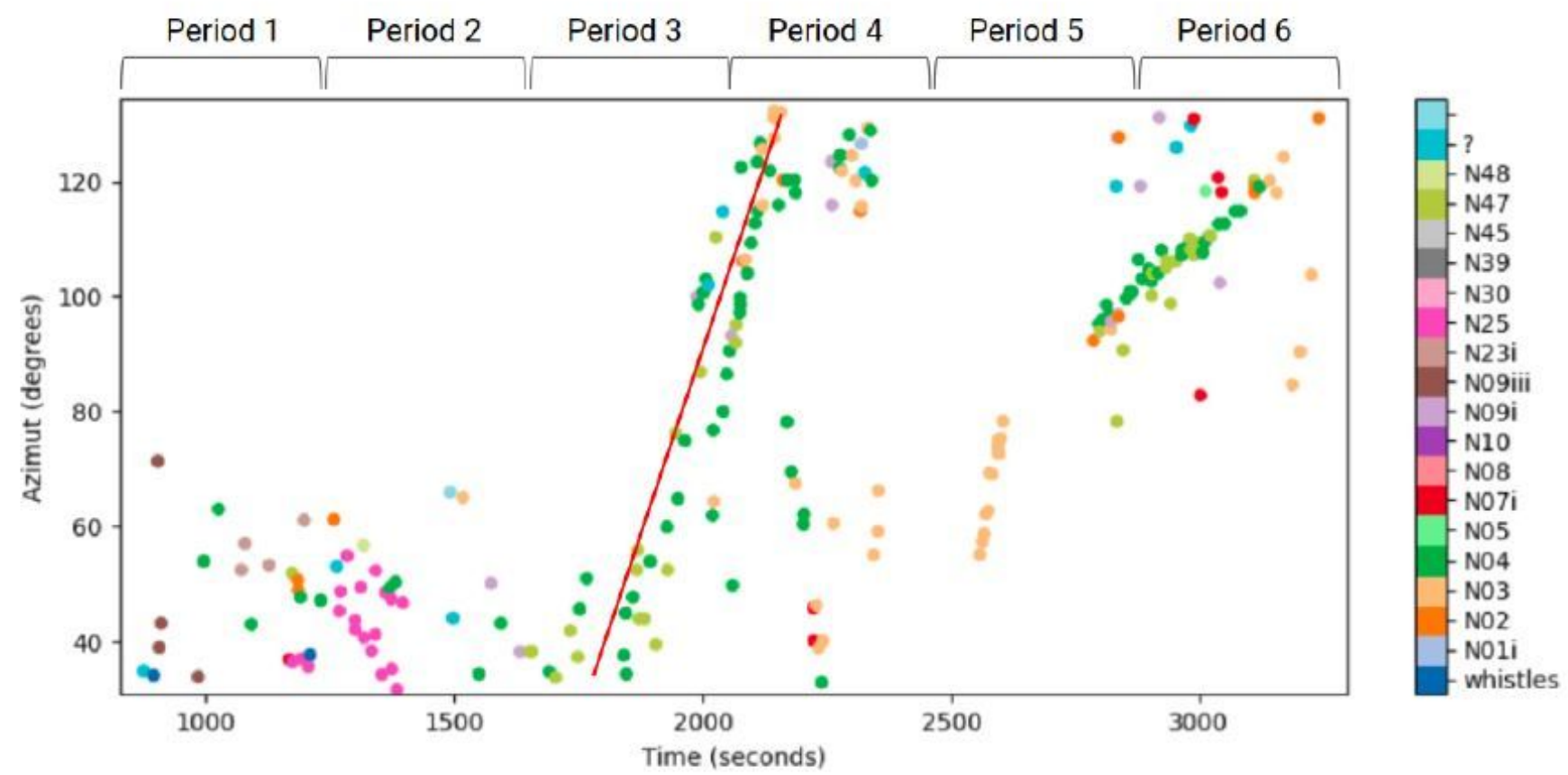

Figure 4

Graph of the passage of the 11th of August. Each point represents a vocalization placed in azimuth. Each color represents a type of call. The most common types are N04, N03, N48. The red line is the visual trajectory of the boat. 


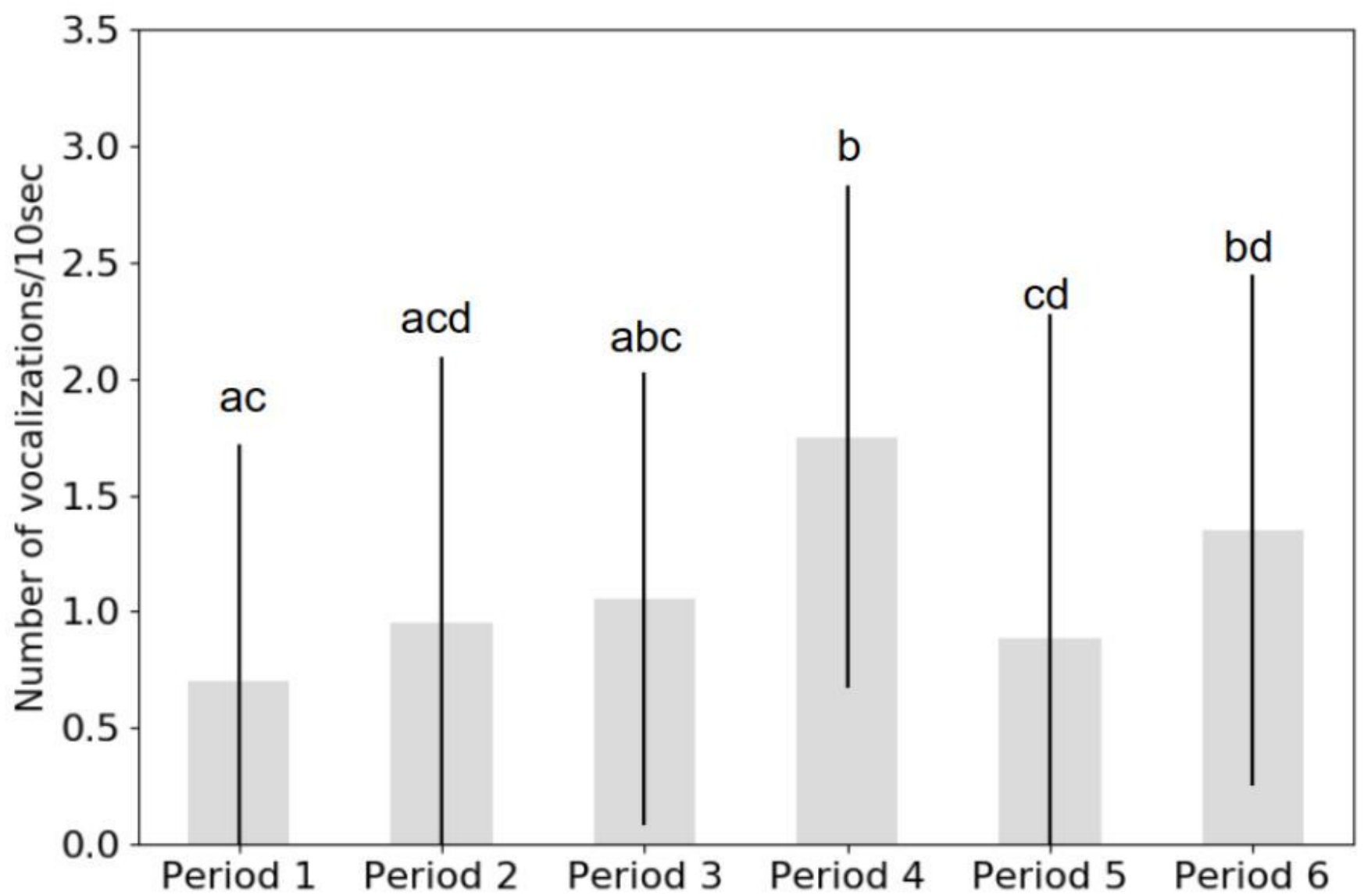

Figure 5

Graph of the CR of the 11st of August for each period (1 to 6). 


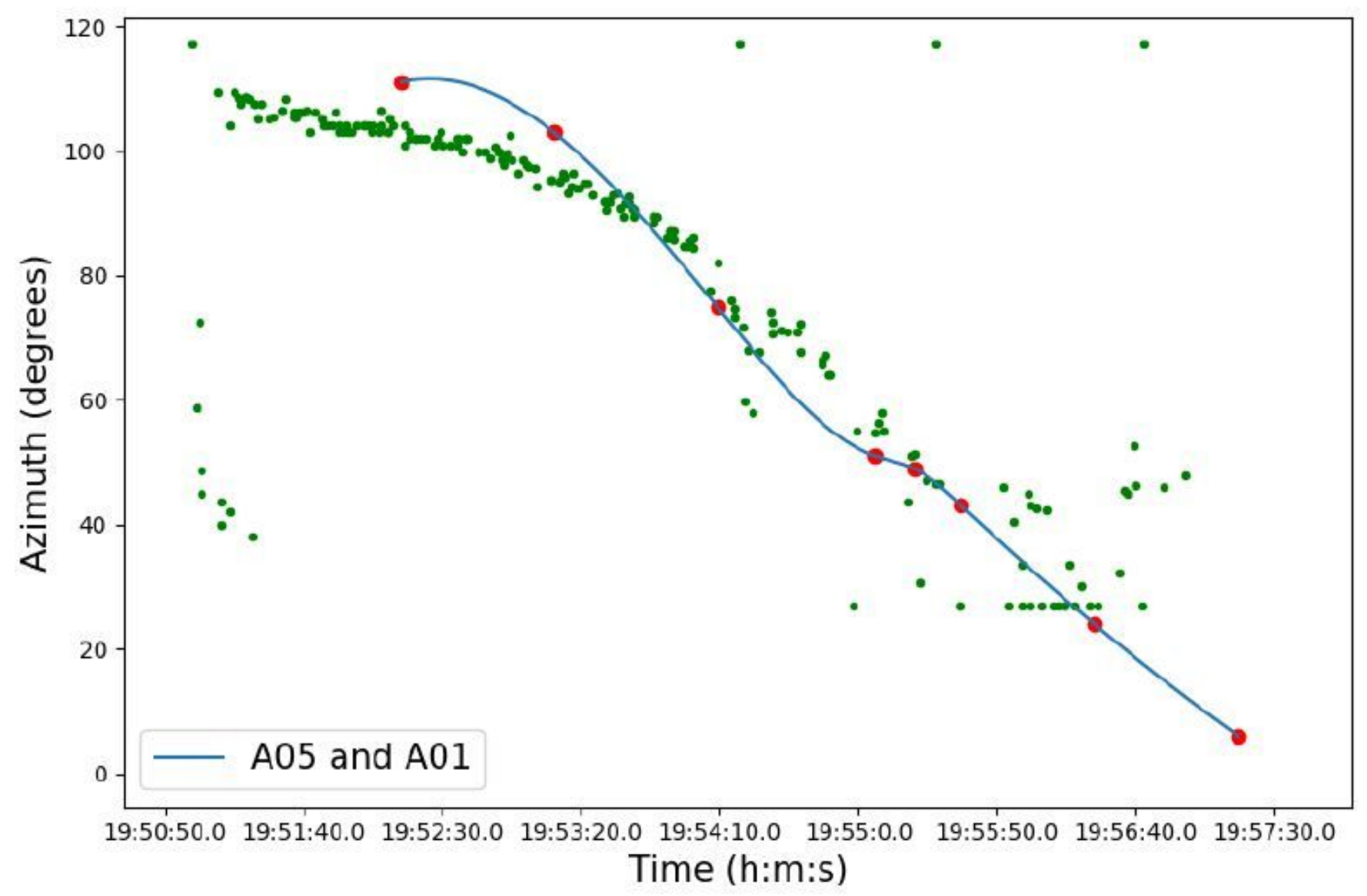

Figure 6

Graph of the passage of the 30th of August. Each green point represents a vocalization placed in azimuth. The blue curve is the interpolation of the A05 and A01 visual azimuth estimations. 


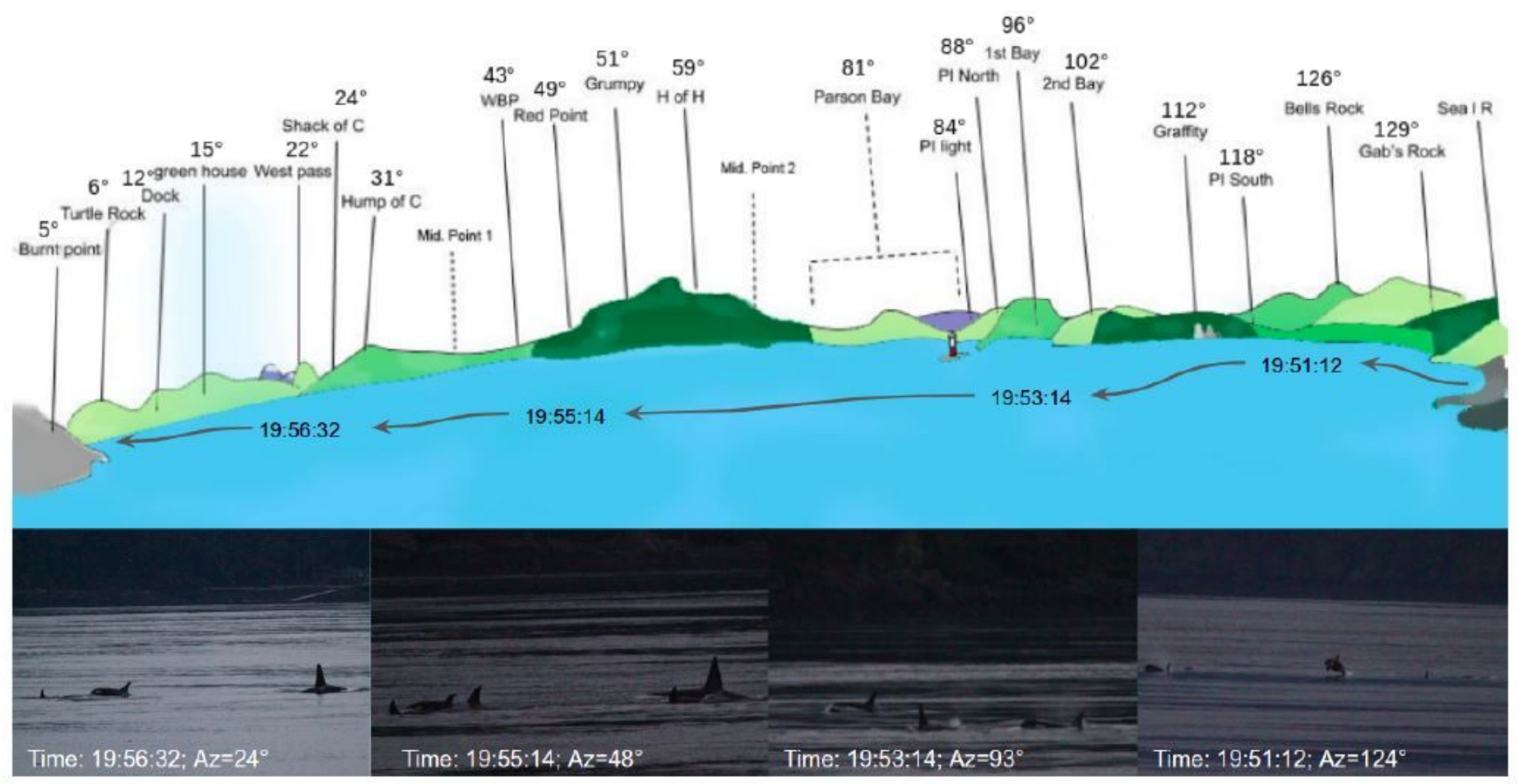

\section{Figure 7}

Graph of the passage of the 30st of August. Top : representation of the view of BP from OL with the visual position of the group. Bottom : pictures taken from OL deck. 

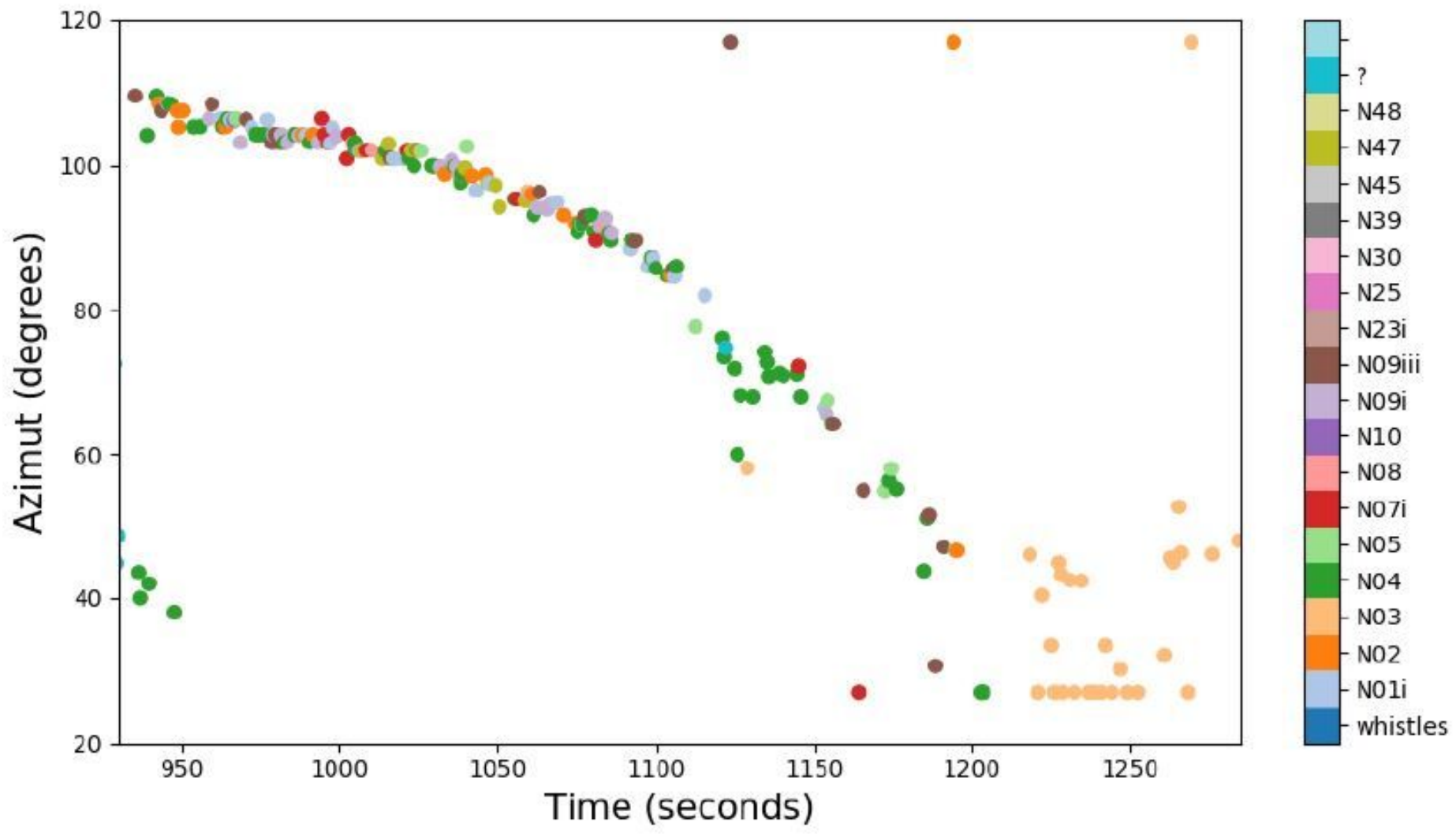

Figure 8

Graph of the passage of the 30th of August. Each point represents a vocalization placed in azimuth. Each color represents a type of call. 


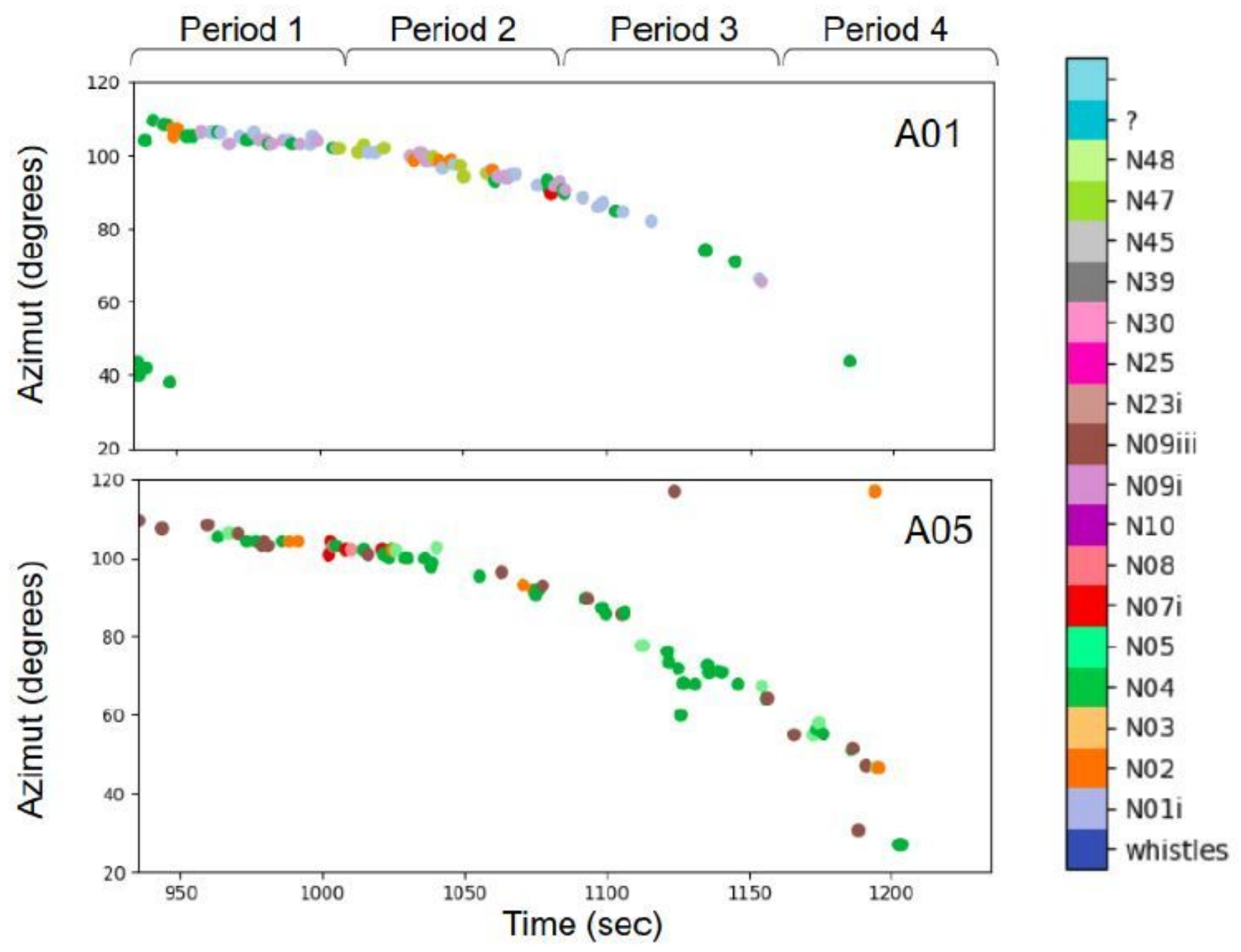

Figure 9

Graph of the passages of the 30th of August. Each point represents a vocalization placed in azimuth. Each color represents a type of call. Top : trajectory of the A01s, Bottom : trajectory of the A05s. 


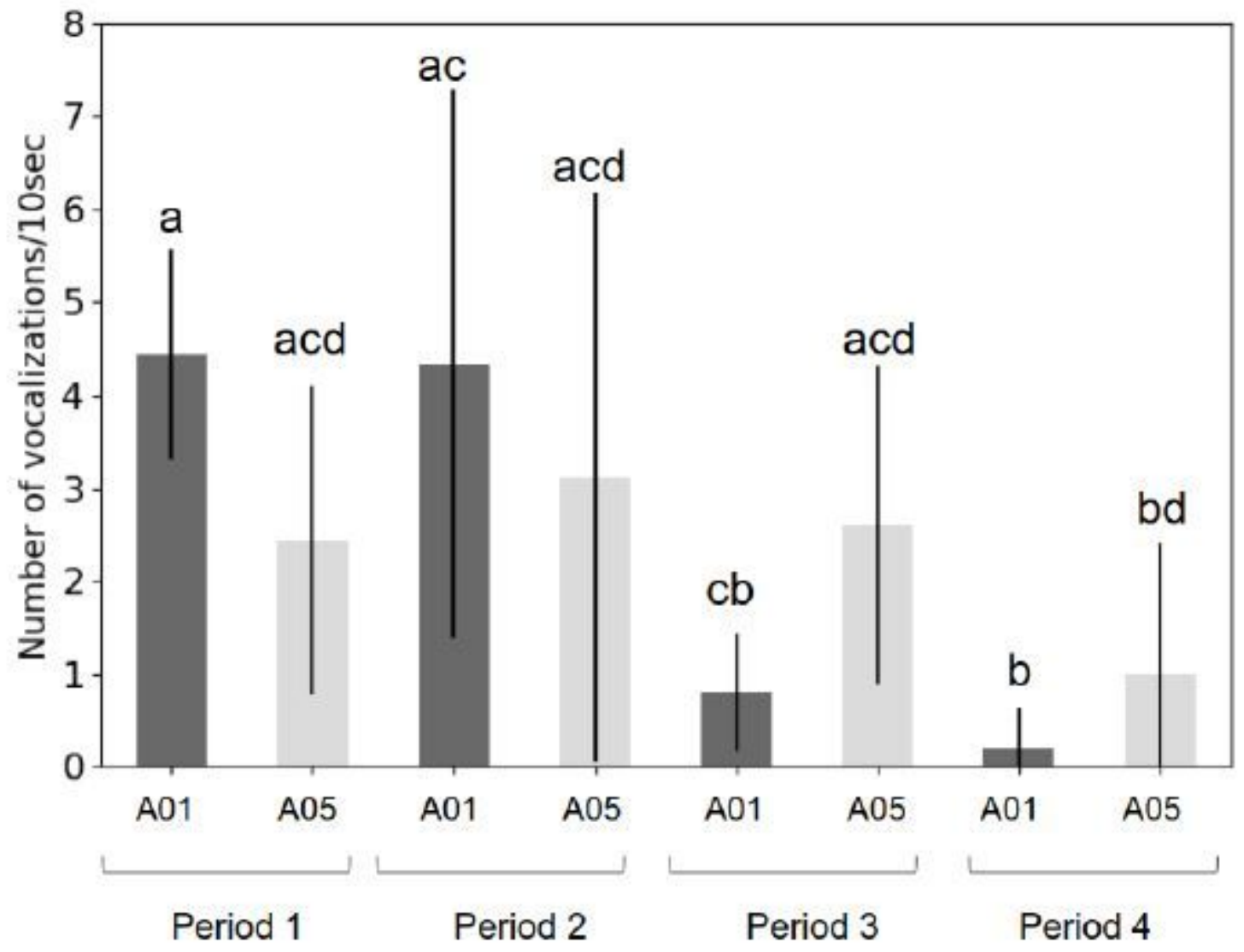

Figure 10

Graph of the CR of the 30st of August for each period and for the A01s (dark grey) and the A05s (light grey). 


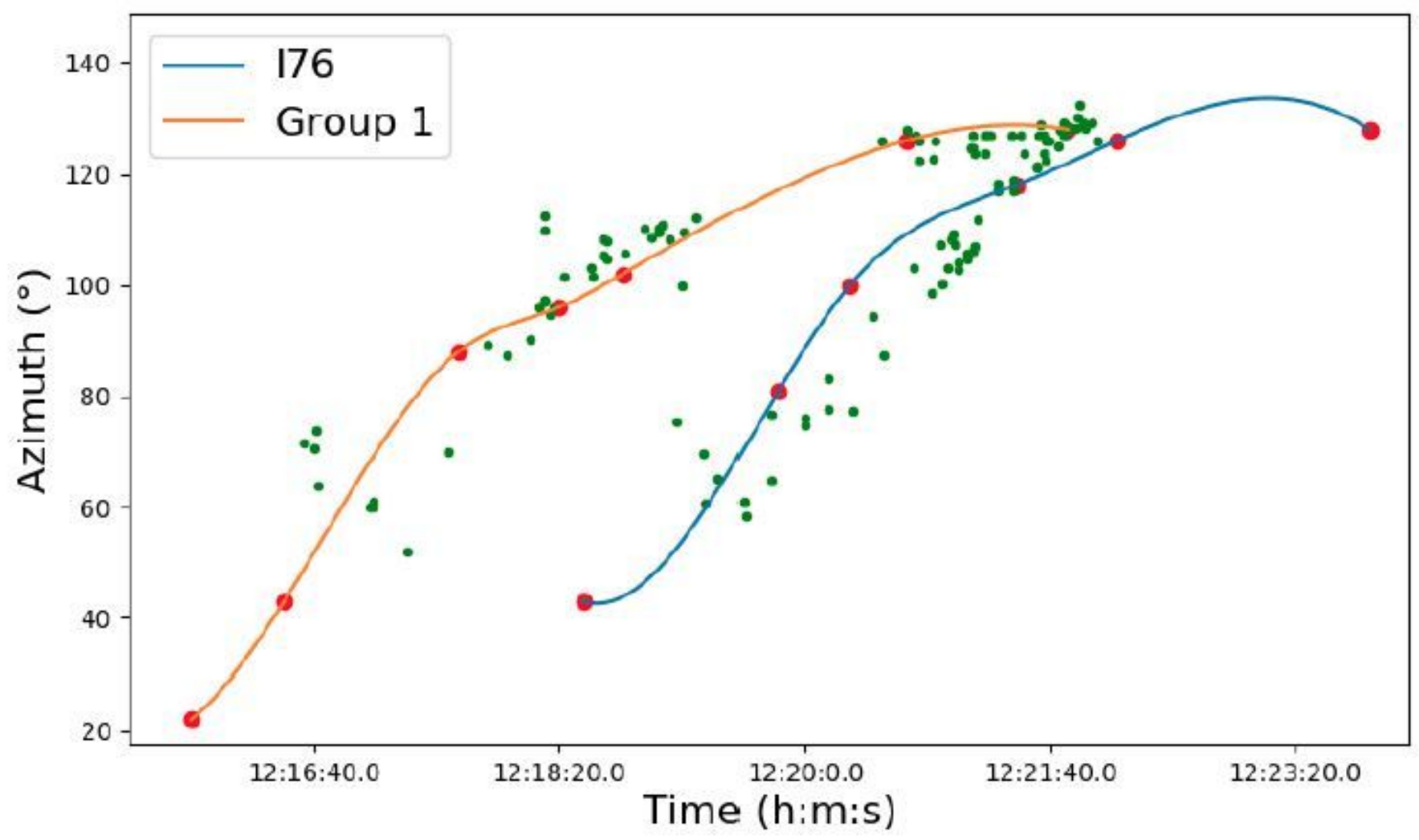

Figure 11

Graph of the passage of the 31 st of August. Each green point represents a vocalization placed in azimuth. The blue curve is the interpolated position of the male 176 visually detected, the orange curve is the large group. 


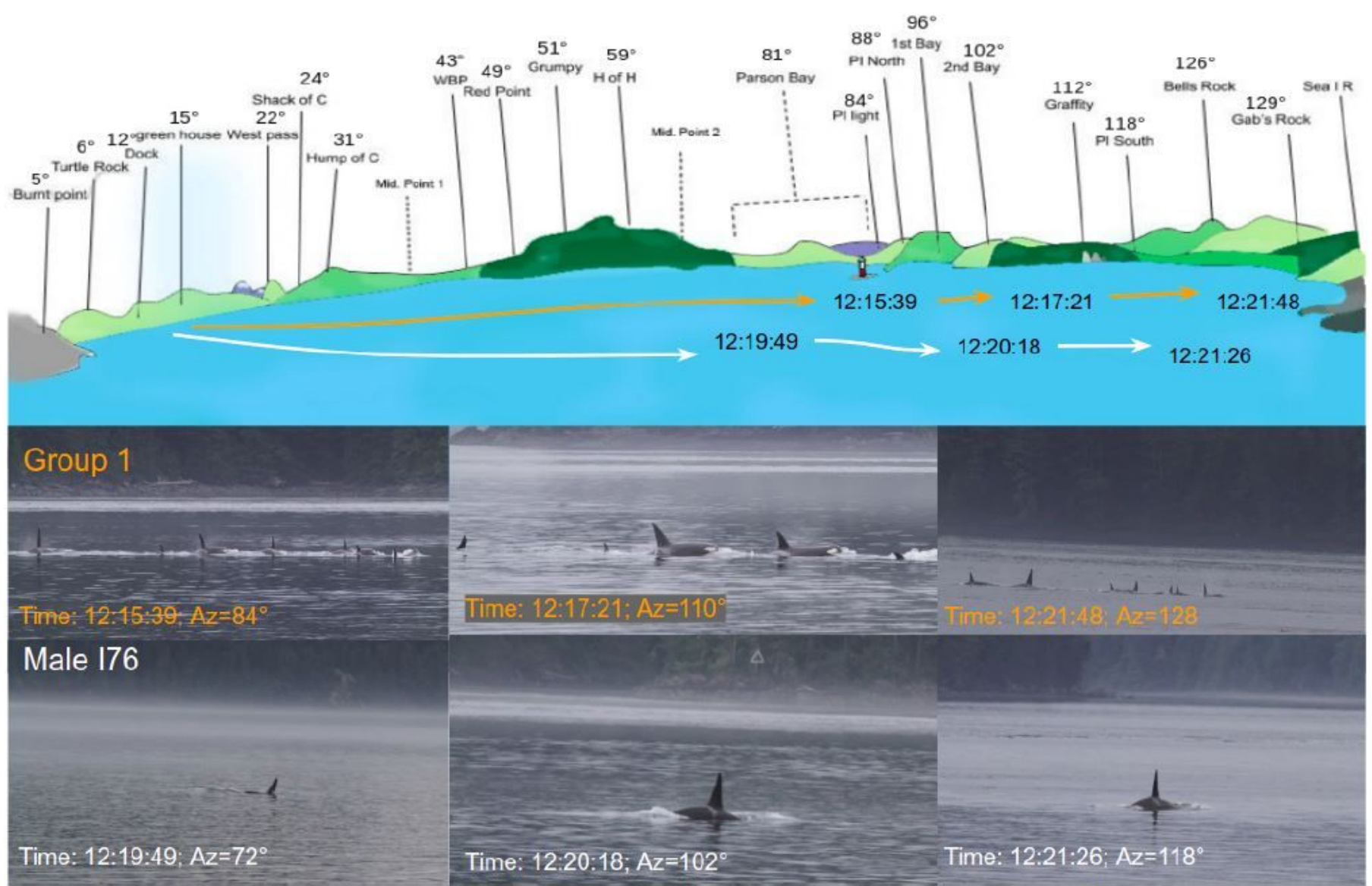

Figure 12

Graph of the passage of the 31st of August. Top : representation of the view of BP from OL with the visual position of the group (orange) and the male 176 (white). Bottom : pictures taken from OL. 


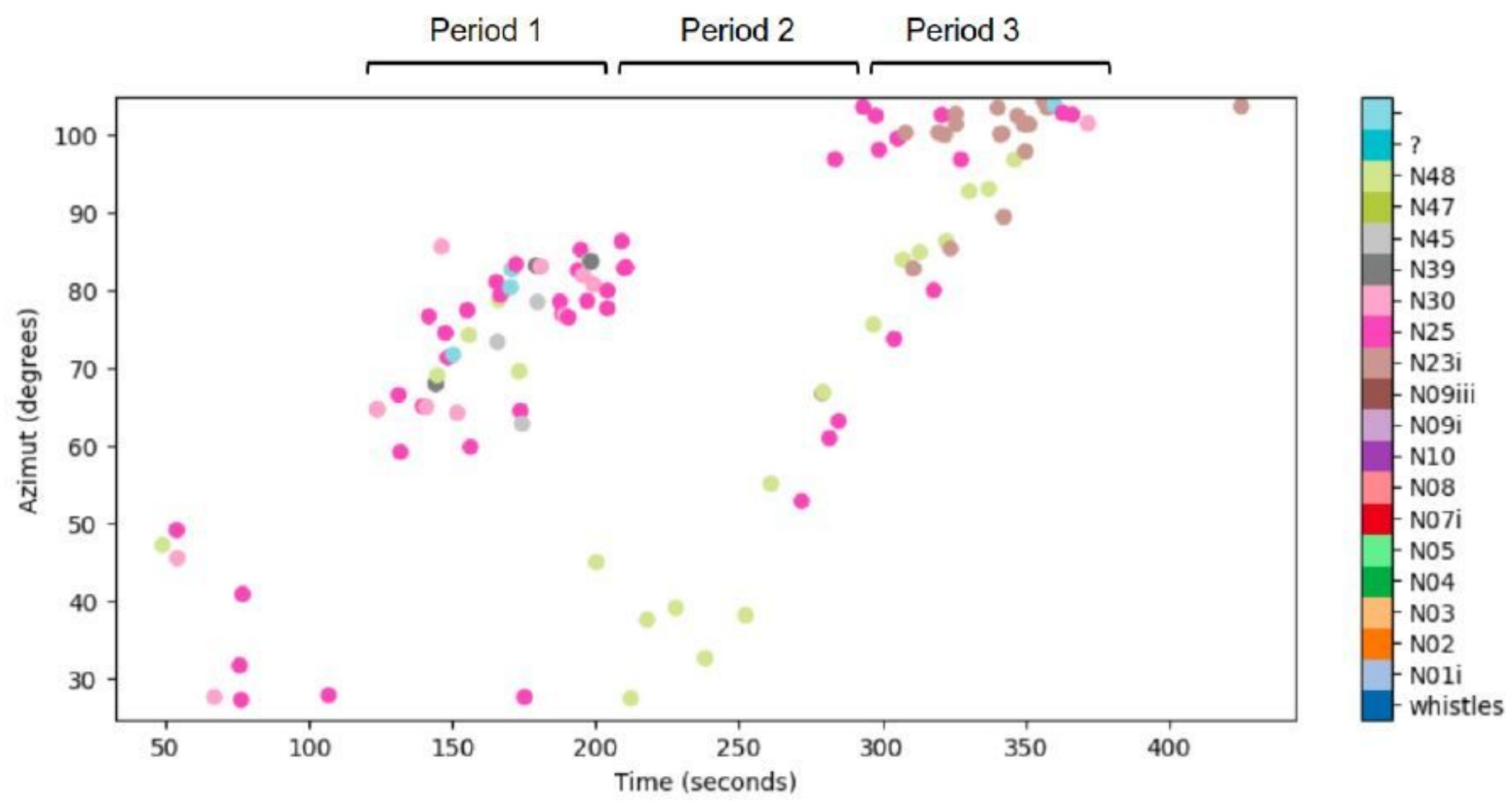

Figure 13

Graph of the passage of the 31 st of August. Each point represents a vocalization placed in azimuth. Each color represents a type of call. The most common types are N25, N48 and N23i. The track was split into three 80 s periods. 


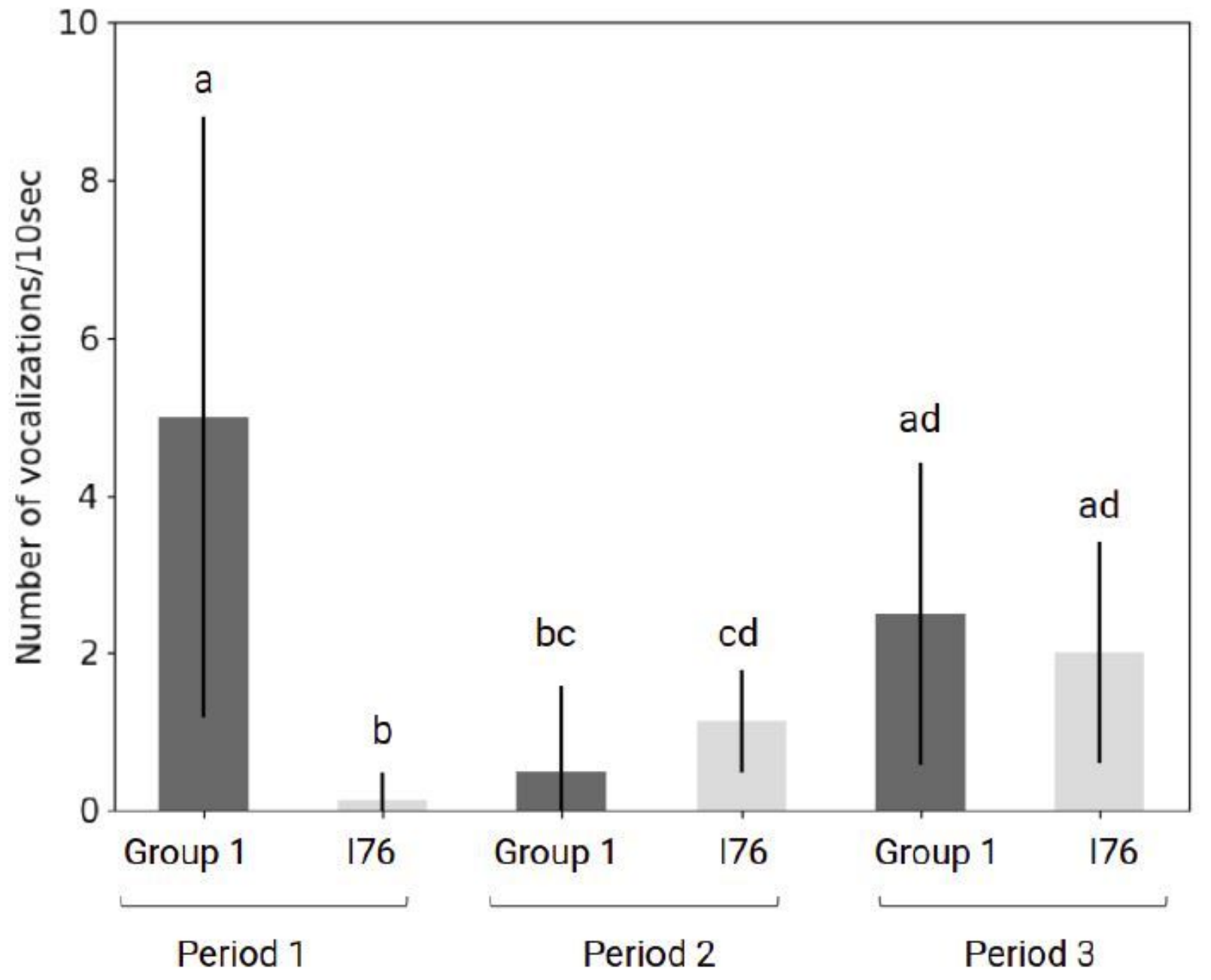

Figure 14

Graph of the CR of the 31st of August for each period and for the group (dark grey) and the male 176 (light grey). 


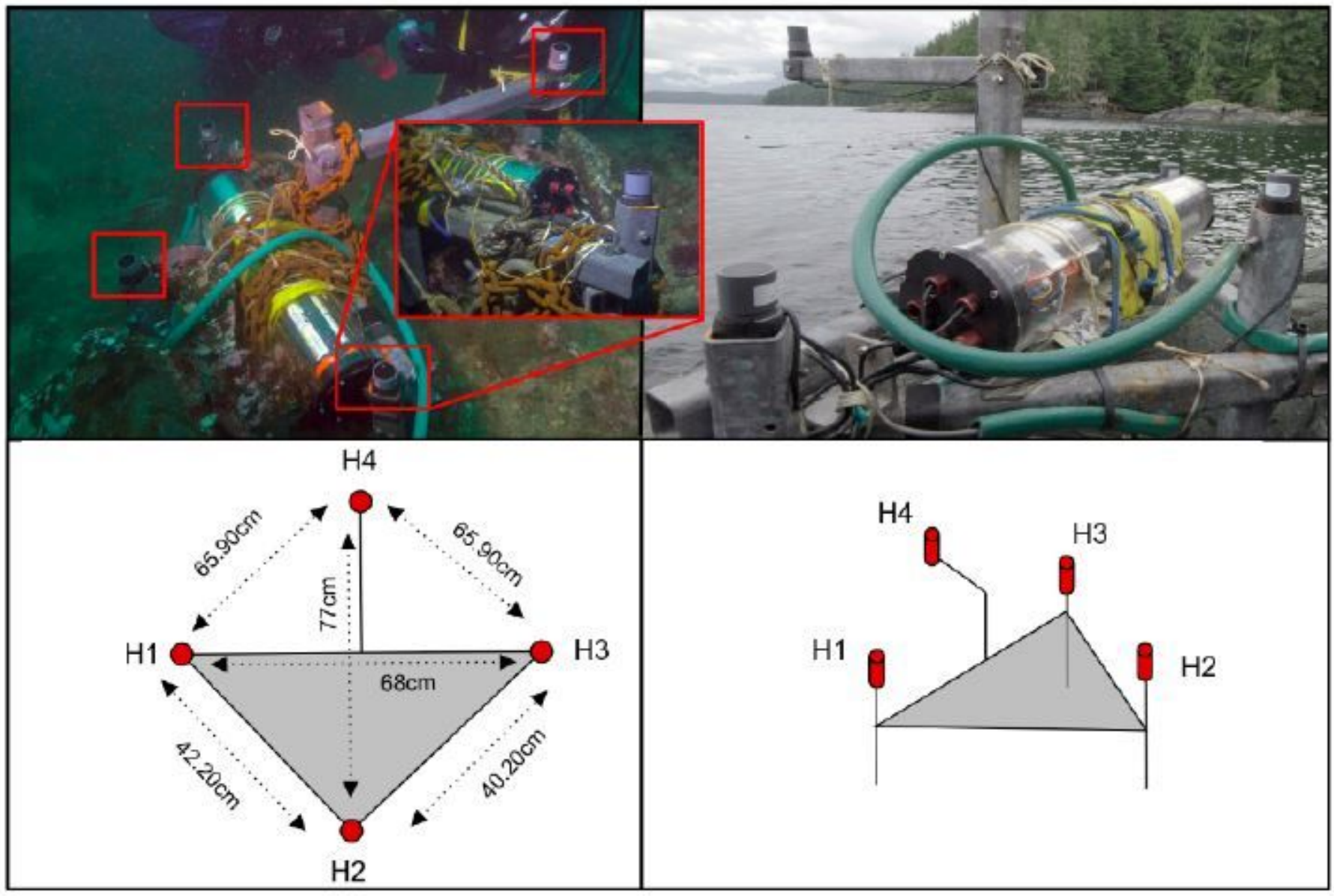

Figure 15

Top : the four-hydrophone antenna, with the $12 \mathrm{~V}$ (green) cable connected into OL. Bottom : diagram of the antenna and its inter-hydrophone distances.

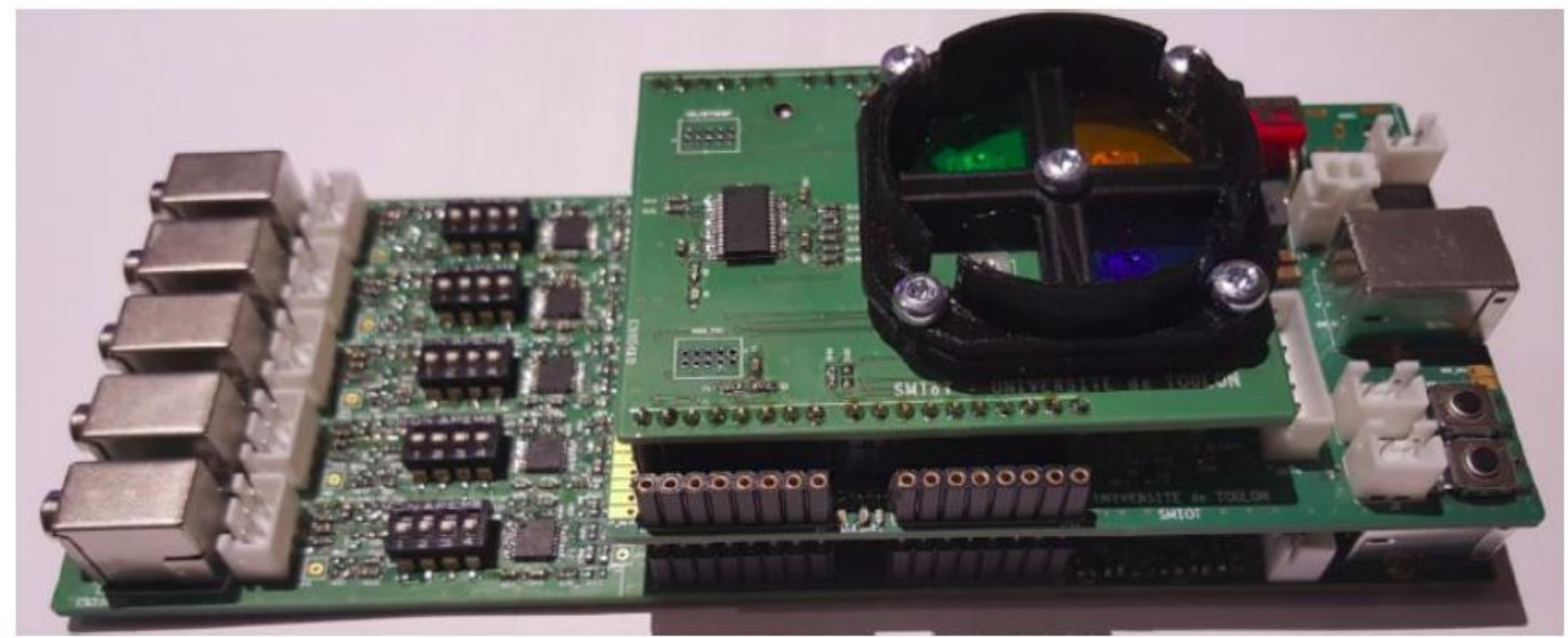




\section{Figure 16}

The JASON sound card $5 \times 2 \mathrm{MHz}$ sampling rate at 16 bits resolution, placed into the submarine tube (the luxmeter was not used here).

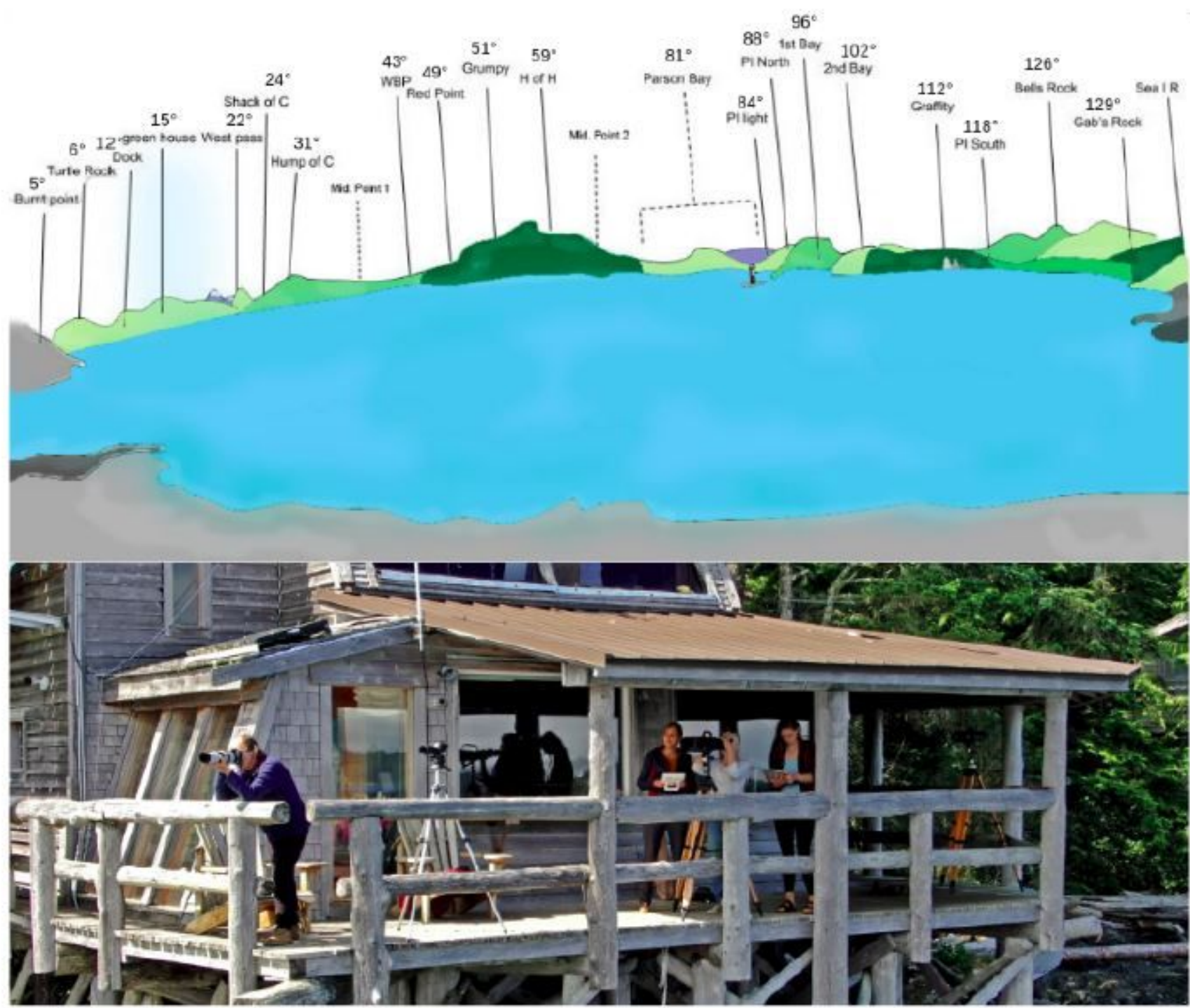

\section{Figure 17}

(Top) Representation of the view of BP from OL. Each site was assigned a precise azimuth using a compass (ex : "Pi Light" = 84 degrees.). (Bottom) Picture of the visual protocol. 


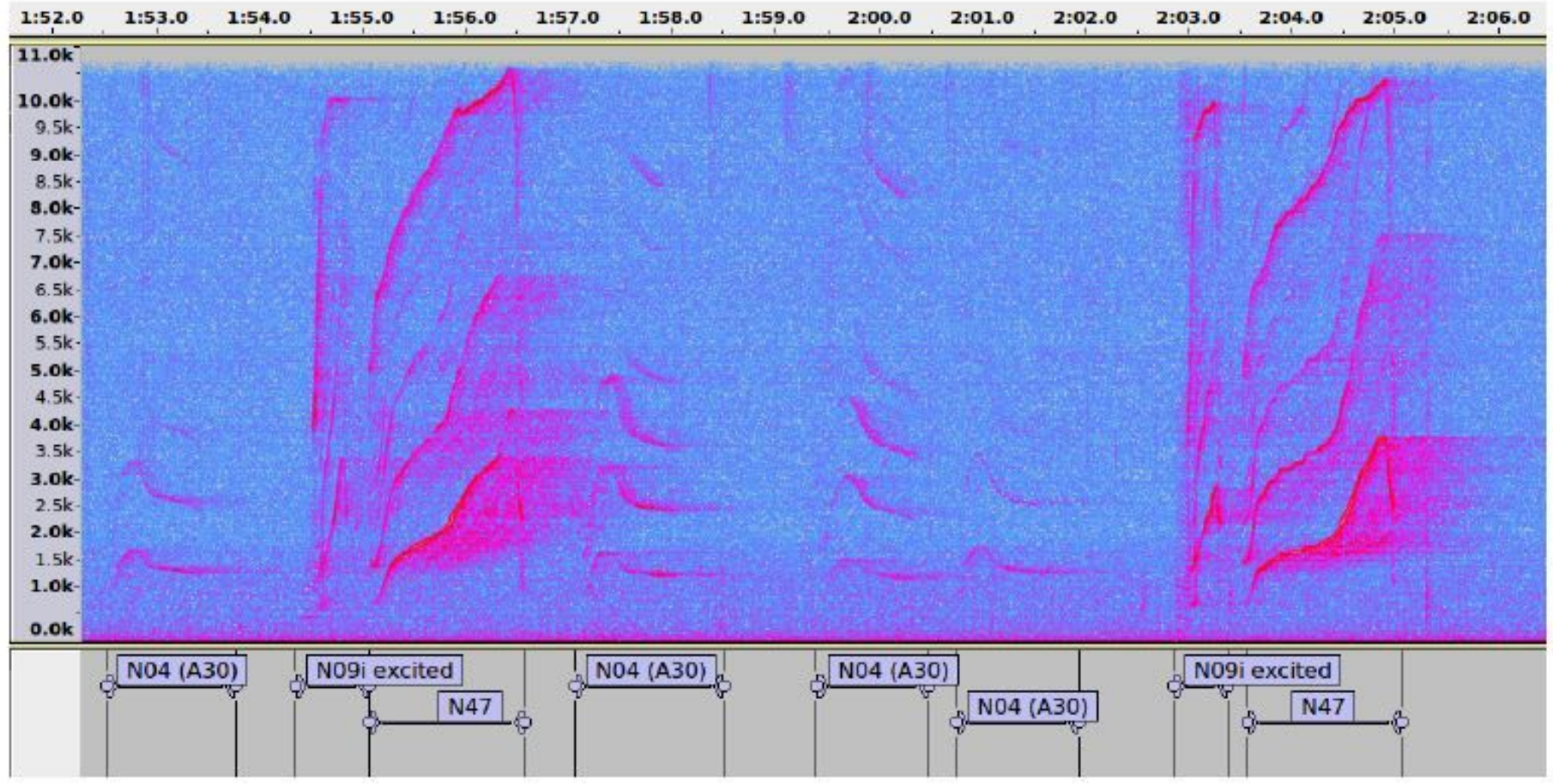

Figure 18

Example of annotation of $13 \mathrm{~s}$ of recordings (Audacity). Hanning window size : 1024. 


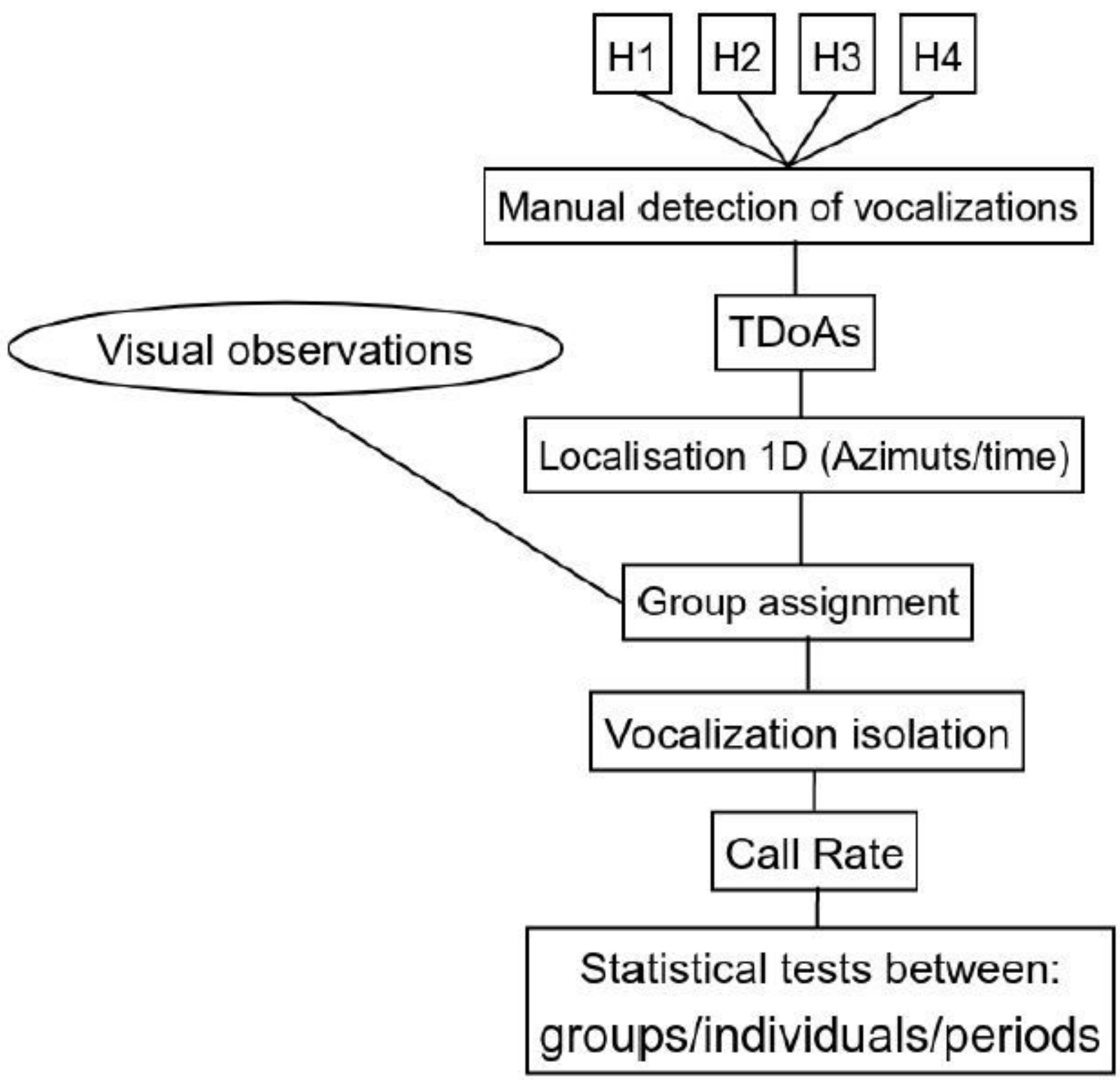

Figure 19

Synopsis of the protocol. 


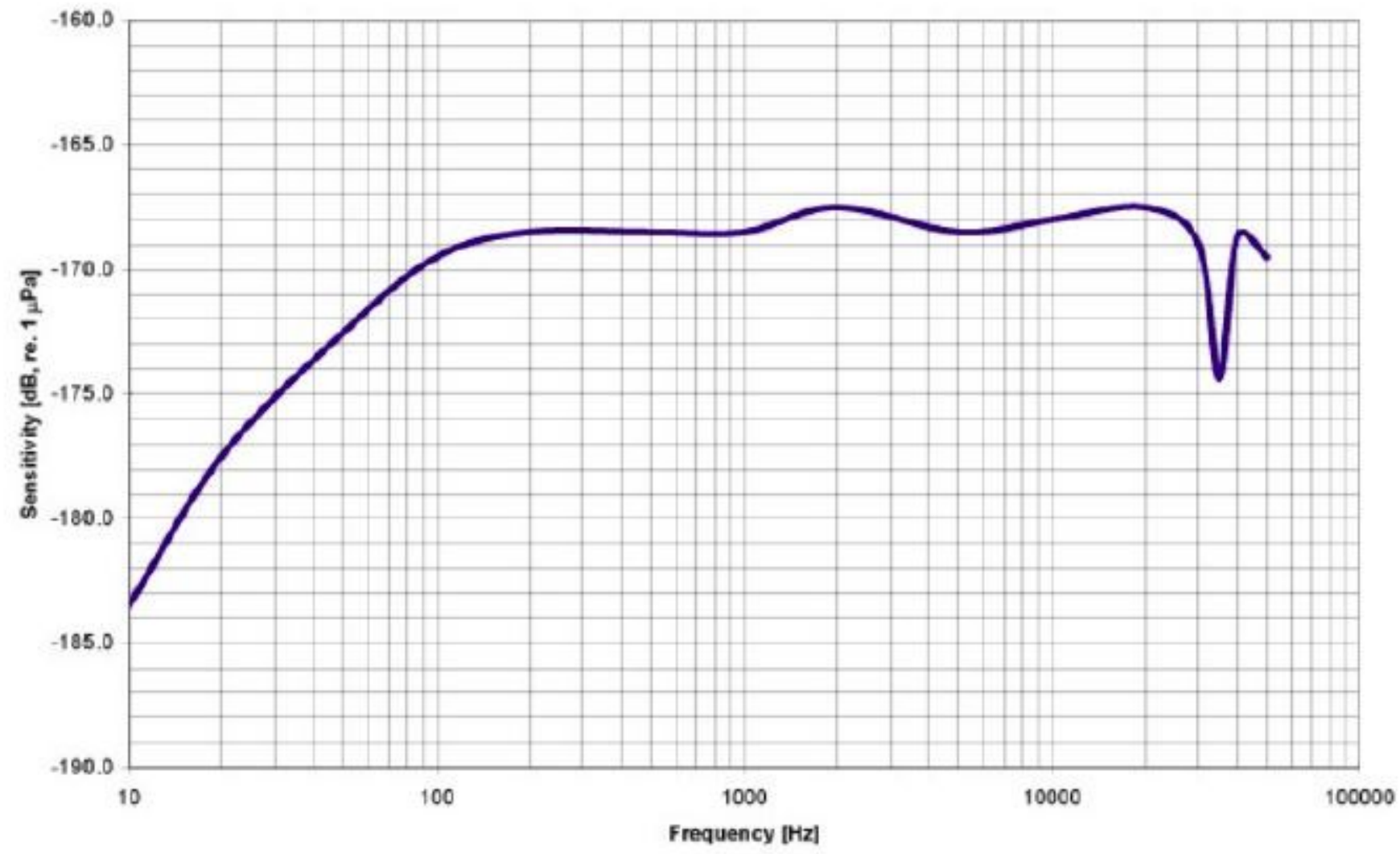

Figure 20

SQ26-07 Frequency response49

\section{Supplementary Files}

This is a list of supplementary files associated with this preprint. Click to download.

- supmaterialrecordingsample.txt 